\author{
Hentosh O.Ye. ${ }^{1}$, BALINSKY A.A. ${ }^{2}$, PryKarPatsKi A.K. ${ }^{3,4}$
}

\title{
THE GENERALIZED CENTRALLY EXTENDED LIE ALGEBRAIC STRUCTURES AND RELATED INTEGRABLE HEAVENLY TYPE EQUATIONS
}

\begin{abstract}
There are studied Lie-algebraic structures of a wide class of heavenly type non-linear integrable equations, related with coadjoint flows on the adjoint space to a loop vector field Lie algebra on the torus. These flows are generated by the loop Lie algebras of vector fields on a torus and their coadjoint orbits and give rise to the compatible Lax-Sato type vector field relationships. The related infinite hierarchy of conservations laws is analysed and its analytical structure, connected with the Casimir invariants, is discussed. We present the typical examples of such equations and demonstrate in details their integrability within the scheme developed. As examples, we found and described new multidimensional generalizations of the Mikhalev-Pavlov and Alonso-Shabat type integrable dispersionless equation, whose seed elements possess a special factorized structure, allowing to extend them to the multidimensional case of arbitrary dimension.

Key words and phrases: heavenly type equations, Lax integrability, Hamiltonian system, torus diffeomorphisms, loop Lie algebra, central extension, Lie-algebraic scheme, Casimir invariants, LiePoisson structure, $R$-structure, Mikhalev-Pavlov equations.
\end{abstract}

\footnotetext{
${ }^{1}$ Pidstryhach Institute for Applied Problems of Mechanics and Mathematics, 3b Naukova str., 79060, Lviv, Ukraine

2 School of Mathematics, Cardiff University, CF24 4AG, Cardiff, Great Britain

${ }^{3}$ Cracow University of Technology, 31-155, Cracow, Poland

${ }^{4}$ Drohobych Ivan Franko State Pedagogical University, 24 Franka str., 82100, Drohobych, Ukraine

E-mail: ohen@ukr.net (Hentosh O.Ye.), BalinskyA@cardiff.ac.uk (Balinsky A.A.), pryk . anat@cybergal. com (Prykarpatski A.K.)
}

\section{INTRODUCTION}

The main object of our study are integrable multidimensional dispersionless differential equations, which possess modified Lax-Sato type representations, related with their hidden Hamiltonian structures. Equations of this type arise and widely applied in mechanics, general relativity, differential geometry and the theory of integrable systems. Among the most one can mention the Boyer-Finley equation, heavenly type Plebański equations, which are descriptive of a class of self-dual four-manifolds, as well as the dispersionless KadomtsevPetviashvili (dKP) equation, also known as the Khokhlov-Zabolotskaya equation, which arises in non-linear acoustics and the theory of Einstein-Weyl structures. Their integrability have been investigated by a whole variety of modern techniques including symmetry analysis, differential-geometric and algebro-geometric methods, dispersionless $\bar{\partial}$-dressing, factorization techniques, Virasoro constraints, hydrodynamic reductions, etc. The first examples and the importance of the related Hamiltonian structures were before demonstrated in $[29,36,38]$ and later were developed in $[25,43]$, where there were analyzed in detail many examples of dispersionless differential equations as flows on orbits of the coadjoint action of loop vector 
field algebras $\widetilde{\operatorname{diff}}\left(\mathbb{T}^{n}\right)$, generated by specially chosen seed elements $\tilde{l} \in \widetilde{\operatorname{dif} f}\left(\mathbb{T}^{n}\right)^{*}$. In these works there was observed that many integrable multidimensional dispersionless differential equations are generated by seed elements of a very special structure, namely for them there exist such analytical functional elements $\tilde{\eta}, \tilde{\rho} \in \Lambda^{0}\left(C^{\infty}\left(\mathbb{T}^{n} ; \mathbb{R}\right)\right) \otimes \mathbb{C}$ that $\tilde{l}=\tilde{\eta} d \tilde{\rho}$. As the latter naturally generates the symplectic structure $\tilde{\omega}^{(2)}:=\int_{\mathbb{T}^{n}} d \tilde{\eta} \wedge d \tilde{\rho} \in \Lambda^{2}\left(\mathbb{T}^{n}\right) \otimes \mathbb{C}$ on the moduli space $[2,42]$ of flat connections on $\mathbb{T}^{n}$, related to coadjoint actions of the corresponding Casimir functionals, the geometric nature of many integrable multidimensional dispersionless differential equations can be also studied using cohomological techniques, devised in $[2,10]$ in the case of Riemannian surfaces. It is worth also to mention a revealed in [25] deep connection of the related Hamiltonian flows on $\widehat{\operatorname{diff}}\left(\mathbb{T}^{n}\right)^{*}$ with the well known in classical mechanics Lagrange-d'Alembert principle.

In this article, in part developing the approach, devised in $[29,38]$, we describe a Lie algebraic structure and integrability properties of a generalized hierarchy of the Lax-Sato type compatible systems of Hamiltonian flows and related integrable multidimensional dispersionless differential equations. Such systems are called the heavenly type equations and were first introduced by Plebański in [41]. The heavenly type equations were analyzed in many articles (see, e.g., [16, 19-22, 32, 38, 39] and [40,46, 47, 52, 53]) using several different approaches. In $[7-9,50]$ the heavenly type equations were analyzed by using nonassociative and noncommutative current algebras on the torus $\mathbb{T}^{m}, m \in \mathbb{N}$. Mention also that $[49,51]$ B. Szablikowski and A. Sergyeyev developed some generalizations of the classical AKS-algebraic and related $R$-structures $[11,13,15,45,54]$. In [38,39] and recently in [25] these ideas were applied to a semi-direct Lie algebra $\left.\mathcal{T}^{n}\right)^{*}$ of the loop Lie algebra $\widetilde{\operatorname{diff}}\left(\mathbb{T}^{n}\right):=\widetilde{\operatorname{Vect}}\left(\mathbb{T}^{n}\right)$ of vector fields on the torus $\mathbb{T}^{n}, n \in \mathbb{Z}_{+}$, and its dual space $\widetilde{\operatorname{diff}}\left(\mathbb{T}^{n}\right)^{*}$. Several interesting and deep results about orbits of the corresponding coadjoint actions on the space $\tilde{\mathcal{G}}^{*} \simeq \tilde{\mathcal{G}}$ and the classical LiePoisson type structures on them were presented. It is worth to specially remark here that the AKS-algebraic scheme is naturally imbedded into the classical $R$-structure approach via the following construction.

Let $(\tilde{\mathcal{G}} ;[\cdot, \cdot])$ denote a Lie algebra over $\mathbb{C}$ and $\tilde{\mathcal{G}}^{*}$ be its natural adjoint space. Take some tensor element $r \in \tilde{\mathcal{G}} \otimes \tilde{\mathcal{G}} \simeq \operatorname{Hom}\left(\tilde{\mathcal{G}}^{*} ; \tilde{\mathcal{G}}\right)$ and consider its splitting into symmetric and antisymmetric parts

$$
r=k \oplus \sigma,
$$

respectively, and assume that the symmetric tensor $k \in \tilde{\mathcal{G}} \otimes \tilde{\mathcal{G}}$ is not degenerate. That allows to define on the Lie algebra $\tilde{\mathcal{G}}$ a symmetric nondegenerate bi-linear product $(\cdot \mid \cdot): \tilde{\mathcal{G}} \otimes \tilde{\mathcal{G}} \rightarrow \mathbb{C}$ via the expression

$$
(a \mid b):=k^{-1} a(b)
$$

for any $a, b \in \tilde{\mathcal{G}}$. The composed mapping $R:=\sigma \circ k^{-1}: \tilde{\mathcal{G}} \rightarrow \tilde{\mathcal{G}}$, following the scheme $\tilde{\mathcal{G}} \stackrel{k^{-1}}{\rightarrow}$ $\tilde{\mathcal{G}}^{*} \stackrel{\sigma}{\rightarrow} \tilde{\mathcal{G}}$, defines the following $R$-structure on the Lie algebra $\tilde{\mathcal{G}}:$

$$
[a, b]_{R}:=[R a, b]+[a, R b]
$$

for all elements $a, b \in \tilde{\mathcal{G}}$. The following theorem, defining the related Poisson structure [10,12, $45,48]$ on the adjoint space $\tilde{\mathcal{G}}$ holds.

Theorem 1. Let $\alpha, \beta \in \tilde{\mathcal{G}}^{*}$ be arbitrary and define the bracket

$$
\{\alpha, \beta\}:=a d_{r \alpha}^{*} \beta-a d_{r \beta}^{*} \alpha .
$$


Then the bracket (2) is Poisson if and only if the R-structure on the Lie algebra $\tilde{\mathcal{G}}$ defines the Lie structure on $\tilde{\mathcal{G}}$, that is there holds the Yang-Baxter equation

$$
[R a, R b]-R[a, b]_{R}=-[a, b]
$$

for any $a, b \in \tilde{\mathcal{G}}$.

The above theorem makes it possible to consider the Hamiltonian flows on the coadjoint space $\tilde{\mathcal{G}}^{*}$ as those determined on the Lie algebra $\tilde{\mathcal{G}}$. The latter is exceptionally useful if for the scalar product (1) there exists such a trace-type $\operatorname{Tr}(\cdot)$ symmetric and ad-invariant functional (of Killing type) that

$$
\operatorname{Tr}(a b):=(a \mid b), \quad(a \mid[b, c])=(([a, b] \mid, c)
$$

for any $a, b$ and $c \in \tilde{\mathcal{G}}$. Then any Hamiltonian flow of an element $a \in \tilde{\mathcal{G}}$ is representable in the standard Lax type form

$$
d a / d t=[\nabla(h), a]
$$

where $\nabla(h) \in \tilde{\mathcal{G}}$ is generated by the corresponding Gateaux derivative of the corresponding smooth Hamiltonian function $h \in \mathcal{D}(\tilde{\mathcal{G}})$.

Concerning the loop Lie algebra $\tilde{\mathcal{G}}:=\widetilde{\operatorname{dif} f}\left(\mathbb{T}^{n}\right)$ on the torus $\mathbb{T}^{n}$, it is well known that such a trace-type functional on $\tilde{\mathcal{G}}$ does not exist, thus we need to study the Hamiltonian flows on the adjoint loop space $\tilde{\mathcal{G}}^{*} \simeq \tilde{\Lambda}^{1}\left(\mathbb{T}^{n}\right)$ of meromorphic differential forms on the torus $\mathbb{T}^{n}$ and obtain, as a result, integrable dispersionless differential equations as compatibility conditions for the related loop vector fields, generated by Casimir functionals on $\tilde{\mathcal{G}}^{*}$. This procedure is much more complicated for analysis than the standard one and employs more geometrical tools and considerations about the orbit space structure of the seed elements $\tilde{l} \in \tilde{\mathcal{G}}^{*}$, generating a hierarchy of integrable Hamiltonian flows. The latter, in part, is deeply related to its reduction properties, guaranteeing the existence of nontrivial Casimir invariants on its coadjoint orbits.

By applying and extending these ideas to central extensions of Lie algebras, we construct new classes of commuting Hamiltonian flows on an extended adjoint space $\overline{\mathcal{G}}:=\tilde{\mathcal{G}}^{*} \oplus \mathbb{C}$. These Hamiltonian flows are generated by seed elements $(\tilde{a} \ltimes \tilde{l} ; \alpha) \in \overline{\mathcal{G}}^{*}$ and specially constructed Casimir invariants on the corresponding orbits of $\tilde{\mathcal{G}}^{*}$. In most cases these seed elements appeared to be represented as specially factorized differential objects, whose real geometric nature is still much hidden and not clear. Moreover, we found that the corresponding compatibility condition of constructed Hamiltonian flows coincides exactly with the compatibility condition for a system of related three Lax-Sato type linear vector field equations. As examples, we found and described new multidimensional generalizations of the Mikhalev-Pavlov and Alonso-Shabat type integrable dispersionless equation, whose seed elements possess a special factorized structure, allowing to extend them to the multidimensional case of arbitrary dimension.

\section{DIFFEOMORPHISMS GROUP $\operatorname{Dif} f\left(\mathbb{T}^{n}\right)$ AND ITS DESCRIPTION}

Consider the $n$-dimensional torus $\mathbb{T}^{n}$ and call points $X \in \mathbb{T}^{n}$ as the Lagrangian variables of a configuration $\eta \in \operatorname{Diff}\left(\mathbb{T}^{n}\right)$. The manifold $\mathbb{T}^{n}$, thought of as the target space of a configuration $\eta \in \operatorname{Diff}\left(\mathbb{T}^{n}\right)$, is called the spatial or Eulerian configuration, whose points, called spatial or Eulerian points, will be denoted by small letters $x \in \mathbb{T}^{n}$. Then any one-parametric 
configuration of $\operatorname{Diff}\left(\mathbb{T}^{n}\right)$ is a time $t \in \mathbb{R}$ dependent family $[1,4,6,28,34]$ of diffeomorphisms written as

$$
\mathbb{T}^{n} \ni x=\eta(X, t):=\eta_{t}(X) \in \mathbb{T}^{n}
$$

for any initial configuration $X \in \mathbb{T}^{n}$ and some mappings $\eta_{t} \in \operatorname{Diff}\left(\mathbb{T}^{n}\right), t \in \mathbb{R}$.

Being interested in studying flows on the space of Lagrangian configurations $\eta \in \operatorname{Diff}\left(\mathbb{T}^{n}\right)$ with respect to the temporal variable $t \in \mathbb{R}$, which are generated by group diffeomorphisms $\eta_{t} \in \operatorname{Diff}\left(\mathbb{T}^{n}\right), t \in \mathbb{R}$, let us proceed to describing the structure of tangent $T_{\eta_{t}}\left(\operatorname{Diff}\left(\mathbb{T}^{n}\right)\right)$ and cotangent $T_{\eta_{t}}^{*}\left(\operatorname{Diff}\left(\mathbb{T}^{n}\right)\right)$ spaces to the diffeomorphism group $\operatorname{Diff}\left(\mathbb{T}^{n}\right)$ at the points $\eta_{t} \in \operatorname{Diff}\left(\mathbb{T}^{n}\right)$ for any $t \in \mathbb{R}$. Determine first the tangent space $T_{\eta_{t}}\left(\operatorname{Diff}\left(\mathbb{T}^{n}\right)\right)$ to the diffeomorphism group manifold $\operatorname{Diff}\left(\mathbb{T}^{n}\right)$ at point $\eta \in \operatorname{Diff}\left(\mathbb{T}^{n}\right)$ for which we will make use of the construction, devised before in $[1,4,27]$. Namely, let $\eta \in \operatorname{Diff}\left(\mathbb{T}^{n}\right)$ be a Lagrangian configuration and try to determine the tangent space $T_{\eta}\left(\operatorname{Diff}\left(\mathbb{T}^{n}\right)\right)$ at $\eta \in \operatorname{Diff}\left(\mathbb{T}^{n}\right)$ as the collection of vectors $\xi_{\eta}:=d \eta_{\tau} /\left.d \tau\right|_{\tau=0}$, where $\mathbb{R} \ni \tau \rightarrow \eta_{\tau} \in \operatorname{Diff}\left(\mathbb{T}^{n}\right),\left.\eta_{\tau}\right|_{\tau=0}=\eta$, is a smooth curve on $\operatorname{Diff}\left(\mathbb{T}^{n}\right)$, and for arbitrary reference point $X \in \mathbb{T}^{n}$ there holds $\xi_{\eta}(X)=d \eta_{\tau}(X) /\left.d \tau\right|_{\tau=0}$. The latter equivalently means that the vectors $\xi_{\eta}(X) \in T_{\eta(X)}\left(\mathbb{T}^{n}\right), X \in \mathbb{T}^{n}$, represent a vector field $\xi: \mathbb{T}^{n} \rightarrow T\left(\mathbb{T}^{n}\right)$ on the manifold $\mathbb{T}^{n}$ for any $\eta \in \operatorname{Diff}\left(\mathbb{T}^{n}\right)$. Thus, the tangent space $T_{\eta}\left(\operatorname{Diff}\left(\mathbb{T}^{n}\right)\right)$ coincides with the set of vector fields on $\mathbb{T}^{n}$ :

$$
T_{\eta}\left(\operatorname{Diff}\left(\mathbb{T}^{n}\right)\right) \simeq\left\{\xi_{\eta} \in \Gamma\left(T\left(\mathbb{T}^{n}\right)\right): \xi_{\eta}(X) \in T_{\xi(X)}\left(\mathbb{T}^{n}\right)\right\}
$$

and similarly, the cotangent space $T_{\eta}^{*}\left(\operatorname{Dif} f\left(\mathbb{T}^{n}\right)\right)$ consists of all one-form densities on $\mathbb{T}^{n}$ over $\eta \in \operatorname{Diff}\left(\mathbb{T}^{n}\right):$

$$
T_{\eta}^{*}\left(\operatorname{Diff}\left(\mathbb{T}^{n}\right)\right)=\left\{\alpha_{\eta} \in \Lambda^{1}\left(\mathbb{T}^{n}\right) \otimes \Lambda^{3}\left(\mathbb{T}^{n}\right): \alpha_{\eta}(X) \in T_{\eta(X)}^{*}\left(\mathbb{T}^{n}\right) \otimes\left|\Lambda^{3}\left(\mathbb{T}^{n}\right)\right|\right\}
$$

subject to the canonical nondegenerate pairing $(\cdot \mid \cdot)_{c}$ on $T_{\eta}^{*}\left(\operatorname{Diff}\left(\mathbb{T}^{n}\right)\right) \times T_{\eta}\left(\operatorname{Diff}\left(\mathbb{T}^{n}\right)\right)$ : if $\alpha_{\eta} \in T_{\eta}^{*}\left(\operatorname{Diff}\left(\mathbb{T}^{n}\right)\right), \xi_{\eta} \in T_{\eta}\left(\operatorname{Diff}\left(\mathbb{T}^{n}\right)\right)$, where

$$
\left.\alpha_{\eta}\right|_{X}=\left\langle\alpha_{\eta}(X) \mid d x\right\rangle \otimes d^{3} X,\left.\quad \xi_{\eta}\right|_{X}=\left\langle\xi_{\eta}(X) \mid \partial / \partial x\right\rangle,
$$

then

$$
\left(\alpha_{\eta} \mid \xi_{\eta}\right)_{c}:=\int_{\mathbb{T}^{n}}\left\langle\alpha_{\eta}(X) \mid \xi_{\eta}(X)\right\rangle d^{3} X
$$

The construction above makes it possible to identify the cotangent bundle $T_{\eta}^{*}\left(\operatorname{Diff}\left(\mathbb{T}^{n}\right)\right)$ at the fixed Lagrangian configuration $\eta \in \operatorname{Diff}\left(\mathbb{T}^{n}\right)$ to the tangent space $T_{\eta}\left(\operatorname{Diff}\left(\mathbb{T}^{n}\right)\right)$, as the tangent space $T\left(\mathbb{T}^{n}\right)$ is endowed with the natural internal tangent bundle metric $\langle\cdot \mid \cdot\rangle$ at any point $\eta(X) \in \mathbb{T}^{n}$, identifying $T\left(\mathbb{T}^{n}\right)$ with $T^{*}\left(\mathbb{T}^{n}\right)$ via the related metric isomorphism $\sharp: T^{*}\left(\mathbb{T}^{n}\right) \rightarrow T\left(\mathbb{T}^{n}\right)$. The latter can be also naturally lifted to $T_{\eta}^{*}\left(\operatorname{Diff}\left(\mathbb{T}^{n}\right)\right)$ at $\eta \in \operatorname{Diff}\left(\mathbb{T}^{n}\right)$, namely: for any elements $\alpha_{\eta}, \beta_{\eta} \in T_{\eta}^{*}\left(\operatorname{Diff}\left(\mathbb{T}^{n}\right)\right),\left.\alpha_{\eta}\right|_{X}=\left\langle\alpha_{\eta}(X) \mid d x\right\rangle \otimes d^{3} X$ and $\left.\beta_{\eta}\right|_{X}=$ $\left\langle\beta_{\eta}(X) \mid d x\right\rangle \otimes d^{3} X \in T_{\eta}^{*}\left(\operatorname{Diff}\left(\mathbb{T}^{n}\right)\right)$ we can define the metric

$$
\left(\alpha_{\eta} \mid \beta_{\eta}\right):=\int_{\mathbb{T}^{n}}\left\langle\alpha_{\eta}^{\sharp}(X) \mid \beta_{\eta}^{\sharp}(X)\right\rangle d^{3} X,
$$

where, by definition, $\left.\alpha_{\eta}^{\sharp}(X):=\sharp\left\langle\alpha_{\eta}(X) \mid d x\right\rangle\right), \beta_{\eta}^{\sharp}(X):=\sharp\left\langle\beta_{\eta}(X) \mid d x\right\rangle \in T_{\eta(X)}\left(\mathbb{T}^{n}\right)$ for any $X \in$ $\mathbb{T}^{n}$. Based on the notions above one can proceed to constructing smooth invariant functionals on the cotangent bundle $T^{*}\left(\operatorname{Diff}\left(\mathbb{T}^{n}\right)\right)$ subject to the corresponding co-adjoint actions of the 
diffeomorphism group $\operatorname{Diff}\left(\mathbb{T}^{n}\right)$. Moreover, as the cotangent bundle $T^{*}\left(\operatorname{Diff}\left(\mathbb{T}^{n}\right)\right)$ is a priori endowed with the canonical symplectic structure, equivalent $[1,4,5,11,13,26,30,31,34,45]$ to the corresponding Poisson bracket on the space of smooth functionals on $T^{*}\left(\operatorname{Diff}\left(\mathbb{T}^{n}\right)\right)$, one can study both the related Hamiltonian flows on it and their adjoint symmetries and complete integrability.

Consider now the cotangent bundle $T^{*}\left(\operatorname{Diff}\left(\mathbb{T}^{n}\right)\right)$ as a smooth manifold endowed with the canonical symplectic structure $[1,5]$ on it, equivalent to the corresponding canonical Poisson bracket on the space of smooth functionals on it. Taking into account that the cotangent space $T_{\eta}^{*}\left(\operatorname{Diff}\left(\mathbb{T}^{n}\right)\right)$ at $\eta \in \operatorname{Diff}\left(\mathbb{T}^{n}\right)$, shifted by the right $R_{\eta^{-1}}$ - action to the space $T_{I d}^{*}\left(\operatorname{Diff}\left(\mathbb{T}^{n}\right)\right)$, Id $\in \operatorname{Diff}\left(\mathbb{T}^{n}\right)$, becomes diffeomorphic to the adjoint space $\operatorname{diff} f^{*}\left(\mathbb{T}^{n}\right)$ to the Lie algebra $\operatorname{diff}\left(\mathbb{T}^{n}\right) \simeq \Gamma\left(T\left(\mathbb{T}^{n}\right)\right)$ of vector fields on $\mathbb{T}^{n}$, as there was stated $[34,35,56,57]$ still by $S$. Lie in 1887, this canonical Poisson bracket on $T_{\eta}^{*}\left(\operatorname{Diff}\left(\mathbb{T}^{n}\right)\right)$ transforms $[4,5,24,31,33,34,55-57]$ into the classical Lie-Poisson bracket on the adjoint space $\mathcal{G}^{*}$. Moreover, the orbits of the diffeomorphism group $\operatorname{Diff}\left(\mathbb{T}^{n}\right)$ on $T^{*}\left(\operatorname{Diff}\left(\mathbb{T}^{n}\right)\right)$ respectively transform into the coadjoint orbits on the adjoint space $\mathcal{G}^{*}$, generated by suitable elements of the Lie algebra $\mathcal{G}$. To construct in detail this Lie-Poisson bracket, we formulate preliminary the following simple lemma.

Lemma 1. The Lie algebra $\operatorname{diff}\left(\mathbb{T}^{n}\right) \simeq \Gamma\left(T\left(\mathbb{T}^{n}\right)\right)$ is determined by the following Lie commutator relationships:

$$
\left[a_{1}, a_{2}\right]=\left\langle a_{1} \mid \nabla\right\rangle a_{2}-\left\langle a_{2} \mid \nabla\right\rangle a_{1}
$$

for any vector fields $a_{1}, a_{2} \in \Gamma\left(T\left(\mathbb{T}^{n}\right)\right)$ on the manifold $\mathbb{T}^{n}$.

Proof. Proof of the commutation relationships (3) easily follows from the group multiplication

$$
\left(\varphi_{1, t} \circ \varphi_{2, t}\right)(X)=\varphi_{2, t}\left(\varphi_{1, t}(X)\right)
$$

for any local group diffeomorphisms $\varphi_{1, t}, \varphi_{2, t} \in \operatorname{Diff}\left(\mathbb{T}^{n}\right), t \in \mathbb{R}$, and $X \in \mathbb{T}^{n}$ under condition that $a_{j}(X):=d \varphi_{j, t}(X) /\left.d t\right|_{t=0}$ and $\left.\varphi_{j, t}\right|_{t=0}=\operatorname{Id} \in \operatorname{Diff}\left(\mathbb{T}^{n}\right), j=\overline{1,2}$.

To calculate the Poisson bracket on the cotangent space $T_{\eta}^{*}\left(\operatorname{Diff}\left(\mathbb{T}^{n}\right)\right)$ at any $\eta \in \operatorname{Diff}\left(\mathbb{T}^{n}\right)$, let us consider the cotangent space $T_{\eta}^{*}\left(\operatorname{Diff}\left(\mathbb{T}^{n}\right)\right) \simeq \operatorname{diff} f^{*}\left(\mathbb{T}^{n}\right)$, the adjoint space to the tangent space $T_{\eta}\left(\operatorname{Diff}\left(\mathbb{T}^{n}\right)\right)$ of left invariant vector fields on $\operatorname{Diff}\left(\mathbb{T}^{n}\right)$ at any $\eta \in \operatorname{Diff}\left(\mathbb{T}^{n}\right)$, and take the canonical symplectic structure on $T_{\eta}^{*}\left(\operatorname{Diff}\left(\mathbb{T}^{n}\right)\right)$ in the form $\omega^{(2)}(\mu, \eta):=\delta \alpha(\mu, \eta)$, where the canonical Liouville form $\alpha(\mu, \eta):=(\mu \mid \delta \eta)_{c} \in \Lambda_{(\mu, \eta)}^{1}\left(T_{\eta}^{*}\left(\operatorname{Diff}\left(\mathbb{T}^{n}\right)\right)\right)$ at a point $(\mu, \eta) \in$ $T_{\eta}^{*}\left(\operatorname{Diff}\left(\mathbb{T}^{n}\right)\right)$ is defined a priori on the tangent space $T_{\eta}\left(\operatorname{Diff}\left(\mathbb{T}^{n}\right)\right) \simeq \Gamma(T(M))$ of rightinvariant vector fields on the torus manifold $\mathbb{T}^{n}$. Having calculated the corresponding Poisson bracket of smooth functions $(\mu \mid a)_{c},(\mu \mid b)_{c} \in C^{\infty}\left(T_{\eta}^{*}\left(\operatorname{Diff}\left(\mathbb{T}^{n}\right)\right) ; \mathbb{R}\right)$ on $T_{\eta}^{*}\left(\operatorname{Diff}\left(\mathbb{T}^{n}\right)\right) \simeq$ $\operatorname{diff} f^{*}\left(\mathbb{T}^{n}\right), \eta \in \operatorname{Diff}\left(\mathbb{T}^{n}\right)$, one can formulate the following proposition.

Proposition 1. The Lie-Poisson bracket on the coadjoint space $T_{\eta}^{*}\left(\operatorname{Diff}\left(\mathbb{T}^{n}\right)\right), \eta \in M$, is equal to the expression

$$
\{f, g\}(\mu)=(\mu \mid[\delta g(\mu) / \delta \mu, \delta f(\mu) / \delta \mu])_{c}
$$

for any smooth right-invariant functionals $f, g \in C^{\infty}\left(\mathcal{G}^{*} ; \mathbb{R}\right)$.

Proof. By definition (see $[1,5])$ of the Poisson bracket of smooth functions $(\mu \mid a)_{c},(\mu \mid b)_{c} \in$ $C^{\infty}\left(T_{\eta}^{*}\left(\operatorname{Diff}\left(\mathbb{T}^{n}\right)\right) ; \mathbb{R}\right)$ on the symplectic space $T_{\eta}^{*}\left(\operatorname{Diff}\left(\mathbb{T}^{n}\right)\right)$, it is easy to calculate that

$$
\{\mu(a), \mu(b)\}:=\delta \alpha\left(X_{a}, X_{b}\right)=X_{a}\left(\alpha \mid X_{b}\right)_{c}-X_{b}\left(\alpha \mid X_{a}\right)_{c}-\left(\alpha \mid\left[X_{a}, X_{b}\right]\right)_{c},
$$


where $X_{a}:=\delta(\mu \mid a)_{c} / \delta \mu=a \in \operatorname{diff}\left(\mathbb{T}^{n}\right), X_{b}:=\delta(\mu \mid b)_{c} / \delta \mu=b \in \operatorname{diff}\left(\mathbb{T}^{n}\right)$. Since the expressions $X_{a}\left(\alpha \mid X_{b}\right)_{c}=0$ and $X_{b}\left(\alpha \mid X_{a}\right)_{c}=0$ owing the right-invariance of the vector fields $X_{a}, X_{b} \in T_{\eta}\left(\operatorname{Diff}\left(\mathbb{T}^{n}\right)\right)$, the Poisson bracket (5) transforms into

$$
\left\{(\mu \mid a)_{c},(\mu \mid b)_{c}\right\}=-\left(\alpha \mid\left[X_{a}, X_{b}\right]\right)_{c}=(\mu \mid[b, a])_{c}=\left(\mu \mid\left[\delta(\mu \mid b)_{c} / \delta \mu, \delta(\mu \mid a)_{c} / \delta \mu\right]\right)_{c}
$$

for all $(\mu, \eta) \in T_{\eta}^{*}\left(\operatorname{Diff}\left(\mathbb{T}^{n}\right)\right) \simeq \operatorname{diff} f^{*}\left(\mathbb{T}^{n}\right), \eta \in \operatorname{Diff}\left(\mathbb{T}^{n}\right)$ and any $a, b \in \operatorname{diff}\left(\mathbb{T}^{n}\right)$. The Poisson bracket (5) is easily generalized to

$$
\{f, g\}(\mu)=(\mu \mid[\delta g(\mu) / \delta \mu, \delta f(\mu) / \delta \mu])_{c}
$$

for any smooth functionals $f, g \in C^{\infty}\left(\mathcal{G}^{*} ; \mathbb{R}\right)$, finishing the proof.

Based on the Lie-Poisson bracket (4), one can naturally construct Hamiltonian flows on the adjoint space $\operatorname{diff} f^{*}\left(\mathbb{T}^{n}\right)$ via the expressions

$$
\partial l / \partial t=-a d_{\nabla h(l)}^{*} l
$$

for any element $l \in \operatorname{diff}^{*}\left(\mathbb{T}^{n}\right), t \in \mathbb{R}$, where, by definition, $\left.\frac{d}{d \varepsilon} h(l+\varepsilon m)\right|_{\varepsilon=0}:=(m \mid \nabla h(l))_{\mathcal{c}}$, for some smooth Hamiltonian function $h \in C^{\infty}\left(\operatorname{diff}^{*}\left(\mathbb{T}^{n}\right) ; \mathbb{R}\right)$. If the system possesses enough additional invariants except the Hamiltonian function, one can expect its simplification often reducing to its complete integrability. Below we proceed to developing an effective enough analytical scheme, before suggested in $[25,37]$ for suitably constructed holomorphic loop diffeomorphism groups on tori, allowing to generate infinite hierarchies of such completely integrable Hamiltonian systems on related functional phase spaces.

\section{HEAVENLY TYPE SYSTEMS: THE MODIFIED LIE-ALGEBRAIC INTEGRABILITY SCHEME}

Let $\left.\widetilde{\operatorname{Diff}}+\mathbb{T}^{n}\right), n \in \mathbb{Z}_{+}$, be subgroups of the loop diffeomorphisms group $\widetilde{\operatorname{Diff}}\left(\mathbb{T}^{n}\right):=$ $\left\{\mathbb{C} \supset \mathbb{S}^{1} \rightarrow \operatorname{Diff}\left(\mathbb{T}^{n}\right)\right\}$, holomorphically extended, respectively, on the interior $\mathbb{D}_{+}^{1} \subset \mathbb{C}$ and on the exterior $\mathbb{D}_{-}^{1} \subset \mathbb{C}$ regions of the unit centrally located disk $\mathbb{D}^{1} \subset \mathbb{C}^{1}$ and such that for any $\tilde{g}(\lambda) \in \widetilde{\operatorname{Diff}_{-}}\left(\mathbb{T}^{n}\right), \lambda \in \mathbb{D}_{-}^{1}, \tilde{g}(\infty)=1 \in \operatorname{Diff}\left(\mathbb{T}^{n}\right)$. The corresponding Lie subalgebras $\left.\widetilde{\operatorname{diff}}+\mathbb{T}^{n}\right) \simeq \widetilde{\operatorname{Vect}_{ \pm}}\left(\mathbb{T}^{n}\right)$ of the loop subgroups $\widetilde{\operatorname{Diff}}_{ \pm}\left(\mathbb{T}^{n}\right)$ are vector fields on $\mathrm{S}^{1} \times \mathbb{T}^{n}$, extended holomorphically, respectively, on regions $\mathbb{D}_{ \pm}^{1} \subset \mathbb{C}^{1}$, where for any $\tilde{a}(\lambda) \in \widetilde{\operatorname{diff}} \_\left(\mathbb{T}^{n}\right)$ the value $\tilde{a}(\infty)=0$. The loop Lie algebra splitting $\widetilde{\operatorname{diff}}\left(\mathbb{T}^{n}\right)=\widetilde{\operatorname{diff}}+\left(\mathbb{T}^{n}\right) \oplus \widetilde{\operatorname{diff}}{ }_{-}\left(\mathbb{T}^{n}\right)$ can be naturally identified with a dense subspace of the dual space $\widetilde{\operatorname{diff}}\left(\mathbb{T}^{n}\right)^{*}$ through the pairing

$$
(\tilde{l} \mid \tilde{a}):=\underset{\lambda \in \mathbb{C}}{\operatorname{res}}(l(x ; \lambda) \mid a(x ; \lambda))_{H^{0}}
$$

with respect to the scalar product

$$
(l(x ; \lambda) \mid a(x ; \lambda))_{H^{0}}:=\int_{\mathbb{T}^{n}} d x\langle l(x ; \lambda), a(x ; \lambda)\rangle
$$

on the usual Hilbert space $H^{0}:=L_{2}\left(\mathbb{T}^{n} ; \mathbb{C}^{n}\right)$ for any elements $\tilde{l} \in \widetilde{\operatorname{diff}}\left(\mathbb{T}^{n}\right)^{*}$ and $\tilde{a} \in \widetilde{\operatorname{diff}}\left(\mathbb{T}^{n}\right)$, naturally represented in their reduced canonical form

$$
\begin{aligned}
& \tilde{a}=\sum_{j=1}^{n} a^{(j)}(x ; \lambda) \frac{\partial}{\partial x_{j}}:=\left\langle a(x ; \lambda), \frac{\partial}{\partial x}\right\rangle, \\
& \tilde{l}=\sum_{j=1}^{n} l_{j}(x ; \lambda) d x_{j}:=\langle l(x ; \lambda), d x\rangle,
\end{aligned}
$$


where we have introduced for brevity the gradient operator $\frac{\partial}{\partial x}:=\left(\frac{\partial}{\partial x_{1}}, \frac{\partial}{\partial x_{2}}, \ldots, \frac{\partial}{\partial x_{n}}\right)^{\top}$ in the Euclidean space $\left(E^{n} ;\langle\cdot, \cdot\rangle\right)$. The corresponding Lie commutator $[\tilde{a}, \tilde{b}] \in \widetilde{\operatorname{diff}}\left(\mathbb{T}^{n}\right)$ of any vector fields $\tilde{a}, \tilde{b} \in \widetilde{\operatorname{diff}}\left(\mathbb{T}^{n}\right)$ is calculated the standard way and equals

$$
[\tilde{a}, \tilde{b}]=\tilde{a} \tilde{b}-\tilde{b} \tilde{a}=\left\langle\left\langle a(x ; \lambda), \frac{\partial}{\partial x}\right\rangle b(x ; \lambda), \frac{\partial}{\partial x}\right\rangle-\left\langle\left\langle b(x ; \lambda), \frac{\partial}{\partial x}\right\rangle a(x ; \lambda), \frac{\partial}{\partial x}\right\rangle .
$$

The Lie algebra $\tilde{\mathcal{G}}$ is naturally split into the direct sum of two Lie subalgebras

$$
\widetilde{\operatorname{diff}}\left(\mathbb{T}^{n}\right)=\widetilde{\operatorname{diff}}+\left(\mathbb{T}^{n}\right)_{+} \oplus \widetilde{\operatorname{diff}}-\left(\mathbb{T}^{n}\right),
$$

for which one can identify the following dual spaces:

$$
\widetilde{\operatorname{diff}}+\left(\mathbb{T}^{n}\right)^{*} \simeq \widetilde{\operatorname{diff}}{ }_{-}\left(\mathbb{T}^{n}\right), \quad \widetilde{\operatorname{diff}}{ }_{-}\left(\mathbb{T}^{n}\right)^{*} \simeq \widetilde{\operatorname{diff}}+\left(\mathbb{T}^{n}\right),
$$

where for any $\tilde{l}(\lambda) \in \widetilde{\operatorname{diff}} \_\left(\mathbb{T}^{n}\right)^{*}$ there holds the constraint $\tilde{l}(0)=0$.

Construct now the Lie algebra $\tilde{\mathcal{G}}:=\widetilde{\operatorname{diff}}\left(\mathbb{T}^{n}\right) \ltimes \widetilde{\operatorname{diff}}\left(\mathbb{T}^{n}\right)^{*}$ as the semi-direct sum of the Lie algebra $\widetilde{\operatorname{diff}}\left(\mathbb{T}^{n}\right)$ and its dual space $\widetilde{\operatorname{diff}}\left(\mathbb{T}^{n}\right)^{*}$, whose Lie structure is given by the following expression

$$
\left[\tilde{a}_{1} \ltimes \tilde{l}_{1}, \tilde{a}_{2} \ltimes \tilde{l}_{2}\right]:=\left[\tilde{a}_{1}, \tilde{a}_{2}\right] \ltimes\left(a d_{\tilde{a}_{2}}^{*} \tilde{l}_{1}-a d_{\tilde{a}_{1}}^{*} \tilde{l}_{2}\right)
$$

for any pair of elements $\left(\tilde{a}_{1} \ltimes \tilde{l}_{1}\right),\left(\tilde{a}_{2} \ltimes \tilde{l}_{2}\right) \in \tilde{\mathcal{G}}$, where $a d^{*} \widetilde{\operatorname{diff}}\left(\mathbb{T}^{n}\right): \widetilde{\operatorname{diff}}\left(\mathbb{T}^{n}\right)^{*} \rightarrow \widetilde{\operatorname{diff}}\left(\mathbb{T}^{n}\right)^{*}$, $\left(a d_{\tilde{a}}^{*} \tilde{l} \mid \tilde{b}\right):=(\tilde{l} \mid[\tilde{a}, \tilde{b}])$ for $\tilde{l} \in \widetilde{\operatorname{diff}}\left(\mathbb{T}^{n}\right)^{*}$ and any $\tilde{a}, \tilde{b} \in \widetilde{\operatorname{diff}}\left(\mathbb{T}^{n}\right)$, is the standard coadjoint mapping of the Lie algebra $\widetilde{\operatorname{dif} f}\left(\mathbb{T}^{n}\right)$ on its adjoint space $\widetilde{\operatorname{diff}}\left(\mathbb{T}^{n}\right)^{*}$ with respect to the pairing (6). The Lie algebra $\tilde{\mathcal{G}}$ can be metricized, as it can be endowed with the nondegenerate symmetric product

$$
\left(\tilde{a}_{1} \ltimes \tilde{l}_{1} \mid \tilde{a}_{2} \ltimes \tilde{l}_{2}\right):=\left(\tilde{l}_{2} \mid \tilde{a}_{1}\right)+\left(\tilde{l}_{1} \mid \tilde{a}_{2}\right),
$$

where $\tilde{a}_{1} \ltimes \tilde{l}_{1}, \tilde{a}_{2} \ltimes \tilde{l}_{2} \in \tilde{\mathcal{G}}$ are arbitrary elements. Owing to the holomorphic structure of the Lie algebra $\widetilde{\operatorname{dif} f}\left(\mathbb{T}^{n}\right)$, the ad-invariant product (8) makes it possible to identify the Lie algebra $\tilde{\mathcal{G}}$ with its dual $\tilde{\mathcal{G}}^{*}$, that is $\tilde{\mathcal{G}}^{*} \simeq \tilde{\mathcal{G}}$. Moreover, the Lie algebra $\tilde{\mathcal{G}}$ can be naturally split $[38,39,49]$ with respect to the pairing (6) and the Lie bracket (7) into two subalgebras $\tilde{\mathcal{G}}=\tilde{\mathcal{G}}_{+} \oplus \tilde{\mathcal{G}}_{-}$, where, by definition,

$$
\tilde{\mathcal{G}}_{+}:=\widetilde{\operatorname{diff}}\left(\mathbb{T}^{n}\right)_{+} \ltimes \widetilde{\operatorname{diff}}\left(\mathbb{T}^{n}\right)_{-}^{*}, \quad \tilde{\mathcal{G}}_{-}:=\widetilde{\operatorname{diff}}\left(\mathbb{T}^{n}\right)_{-} \ltimes \widetilde{\operatorname{diff}}\left(\mathbb{T}^{n}\right)_{+}^{*}
$$

The latter allows to define on the Lie algebra $\tilde{\mathcal{G}}$ a new Lie bracket

$$
\left[\tilde{w}_{1}, \tilde{w}_{2}\right]_{\mathcal{R}}:=\left[\mathcal{R} \tilde{w}_{1}, \tilde{w}_{2}\right]+\left[\tilde{w}_{1}, \mathcal{R} \tilde{w}_{2}\right]
$$

for any elements $\tilde{w}_{1}, \tilde{w}_{2} \in \tilde{\mathcal{G}}$, where $R:=\left(P_{+}-P_{-}\right) / 2$ is the standard $R$-matrix homomorphism $[11,14,44,54]$ on $\tilde{\mathcal{G}}$ and, by definition, $P_{ \pm}: \tilde{\mathcal{G}} \rightarrow \tilde{\mathcal{G}}_{ \pm} \subset \tilde{\mathcal{G}}$ are projectors. The construction above makes it possible to apply to the Lie algebra $\tilde{\mathcal{G}}$ the classical AKS-scheme and, respectively, to generate a wide class of completely integrable Hamiltonian systems as the commuting flows on the adjoint space $\tilde{\mathcal{G}}^{*} \simeq \tilde{\mathcal{G}}$, generated by the corresponding hierarchies of the Casimir invariants subject to the basic Lie bracket (7).

To describe this scheme in more details, we need to find the corresponding Casimir functionals $h \in I\left(\tilde{\mathcal{G}}^{*}\right)$, satisfying, by definition, the following relationship:

$$
a d_{\nabla h(\tilde{l} ; \tilde{a})}^{*}(\tilde{l} ; \tilde{a})=0
$$


at $(\tilde{l} ; \tilde{a}) \in \tilde{\mathcal{G}}^{*} \simeq \tilde{\mathcal{G}}$, where, by definition, the gradient $\nabla h(\tilde{l} ; \tilde{a}):=\nabla h_{\tilde{l}} \ltimes \nabla h_{\tilde{a}} \in \widetilde{\operatorname{diff}}\left(\mathbb{T}^{n}\right) \ltimes$ $\widetilde{\operatorname{diff}}\left(\mathbb{T}^{n}\right)^{*}=\tilde{\mathcal{G}}$ satisfies the following from (9) differential-algebraic equations:

$$
\left[\nabla h_{\tilde{l}}, \tilde{a}\right]=0, \quad a d_{\nabla h_{\tilde{l}}}^{*} \tilde{l}-a d_{\tilde{a}}^{*} \nabla h_{\tilde{a}}=0
$$

for arbitrarily chosen element $\tilde{a} \ltimes \tilde{l} \in \tilde{\mathcal{G}}$. The equations (10) can be rewritten [25] in details as

$$
\begin{aligned}
& \left\langle\nabla h_{l}, \partial / \partial x\right\rangle a-\langle a, \partial / \partial x\rangle \nabla h_{l}=0, \\
& \left\langle\partial / \partial x, \nabla h_{l}\right\rangle l+\left\langle l,\left(\partial / \partial x \nabla h_{l}\right)\right\rangle-\langle\partial / \partial x, a\rangle \nabla h_{a}-\left\langle\nabla h_{a},(\partial / \partial x a)\right\rangle=0,
\end{aligned}
$$

where we put, by definition, that

$$
\begin{gathered}
\nabla h_{\tilde{l}}:=\left\langle\nabla h_{l}, \partial / \partial x\right\rangle, \quad \tilde{a}:=\langle a, \partial / \partial x\rangle, \\
\tilde{l}:=\langle l, d x\rangle, \quad \nabla h_{\tilde{a}}:=\left\langle\nabla h_{a}, d x\right\rangle .
\end{gathered}
$$

The system of linear equation (11) for a given element $\tilde{a} \ltimes \tilde{l} \in \tilde{\mathcal{G}}$, singular as $\lambda \rightarrow \infty$, can be, in general, resolved by means of the asymptotical expressions

$$
\nabla h_{l} \sim \sum_{j \in \mathbb{Z}_{+}} \nabla h_{l}^{(j)} \lambda^{-j}, \quad \nabla h_{a} \sim \sum_{j \in \mathbb{Z}_{+}} \nabla h_{a}^{(j)} \lambda^{-j},
$$

giving rise to an infinite hierarchy of gradients $\nabla h^{(p)}(\tilde{a}, \tilde{l})=\lambda^{p} \nabla h(\tilde{a}, \tilde{l}) \in \tilde{\mathcal{G}}, p \in \mathbb{Z}_{+}$, for the corresponding Casimir functionals $h^{(p)} \in I\left(\tilde{\mathcal{G}}^{*}\right), p \in \mathbb{Z}_{+}$. Similarly, if a given element $\tilde{a} \ltimes \tilde{l} \in \tilde{\mathcal{G}}$ is chosen to be singular as $\lambda \rightarrow 0$, the system of linear equations (11) can be resolved by means of the asymptotical expressions

$$
\nabla h_{l} \sim \sum_{j \in \mathbb{Z}_{+}} \nabla h_{l}^{(j)} \lambda^{j}, \quad \nabla h_{a} \sim \sum_{j \in \mathbb{Z}_{+}} \nabla h_{a}^{(j)} \lambda^{-j}
$$

also generating an infinite hierarchy of gradients $\nabla h^{(p)}(\tilde{l}, \tilde{a})=\lambda^{-p} \nabla h(\tilde{a}, \tilde{l}) \in \tilde{\mathcal{G}}, p \in \mathbb{Z}_{+}$, for the corresponding Casimir functionals $h^{(p)} \in I\left(\tilde{\mathcal{G}}^{*}\right), p \in \mathbb{Z}_{+}$.

Let us now assume that we have already found the gradients $\nabla h^{(y)}(\tilde{a}, \tilde{l}):=\lambda^{p_{y}} \nabla h^{(1)}(\tilde{a}, \tilde{l})$, $\nabla h^{(t)}(\tilde{a}, \tilde{l}):=\lambda^{p_{y}} \nabla h^{(2)}(\tilde{a}, \tilde{l}) \in \tilde{\mathcal{G}}$, related with two Casimir invariants $h^{(1)}, h^{(2)} \in I\left(\tilde{\mathcal{G}}^{*}\right)$ (not necessary different) for some integers $p_{y}, p_{t} \in \mathbb{Z}$, satisfying the determining equations (11). Then, owing to the classical AKS-scheme [11,14,48,54], one can construct two commuting to each other flows with respect to the evolution parameters $y, t \in R$ on the adjoint space $\tilde{\mathcal{G}}^{*} \simeq \tilde{\mathcal{G}}$

$$
\frac{\partial}{\partial y} \tilde{a}=-\left[\nabla h_{\tilde{l},+}^{(y)}, \tilde{a}\right], \quad \frac{\partial}{\partial t} \tilde{a}=-\left[\nabla h_{\tilde{l},+}^{(t)}, \tilde{a}\right]
$$

and

$$
\frac{\partial}{\partial y} \tilde{l}=-a d_{\nabla h_{\tilde{l},+}^{(y)}}^{*} \tilde{l}+a d_{\tilde{a}}^{*}\left(\nabla h_{\tilde{a}_{,+}}^{(y)}\right), \quad \frac{\partial}{\partial t} \tilde{l}=-a d_{\nabla h_{\tilde{l},+}^{(t)}}^{*} \tilde{l}+a d_{\tilde{a}}^{*}\left(\nabla h_{\tilde{a}_{,+}}^{(t)}\right),
$$

where, we have denoted by $\left(\nabla h_{\tilde{l},+}^{(y)} \ltimes \nabla h_{\tilde{a}_{,+}}^{(y)}\right):=P_{+} \nabla h^{(y)}(\tilde{a}, \tilde{l}) \in \tilde{\mathcal{G}}_{+}$and $\left(\nabla h_{\tilde{l},+}^{(t)} \ltimes \nabla h_{\tilde{a}_{,+}}^{(t)}\right):=$ $P_{+} \nabla h^{(t)}(\tilde{a}, \tilde{l}) \in \tilde{\mathcal{G}}_{+}$the corresponding projections on positive degree parts of the corresponding asymptotic expansions (12)-(14). The flows (15) and (16) are, by construction, Hamiltonian, as they are a result of the expressions

$$
\frac{\partial}{\partial y}(\tilde{a} \ltimes \tilde{l})=\left\{\tilde{a} \ltimes \tilde{l}, h^{(y)}\right\}_{\mathcal{R}}, \frac{\partial}{\partial t}(\tilde{a} \ltimes \tilde{l})=\left\{\tilde{a} \ltimes \tilde{l}, h^{(t)}\right\}_{\mathcal{R}}
$$


for a chosen element $\tilde{a} \ltimes \tilde{l} \in \tilde{\mathcal{G}}^{*} \simeq \tilde{\mathcal{G}}$, stemming from the $R$-deformed Lie-Poisson $[11,14,48,54]$ bracket

$$
\{h, f\}_{\mathcal{R}}:=\left(\tilde{a} \ltimes \tilde{l},[\nabla h(\tilde{l}, \tilde{a}), \nabla f(\tilde{l}, \tilde{a})]_{\mathcal{R}}\right)
$$

on the adjoint space $\tilde{\mathcal{G}}^{*} \simeq \tilde{\mathcal{G}}$, defined for any smooth functionals $h, f \in D\left(\tilde{\mathcal{G}}^{*}\right)$. Their commutativity condition is equivalent to two equations such as

$$
\left[\nabla h_{\tilde{l},+}^{(y)}, \nabla h_{\tilde{l},+}^{(t)}\right]-\frac{\partial}{\partial t} \nabla h_{\tilde{l},+}^{(y)}+\frac{\partial}{\partial y} \nabla h_{\tilde{l},+}^{(t)}=0,
$$

and

$$
\begin{gathered}
a d_{\tilde{a}}^{*} \tilde{P}=0, \\
\tilde{P}=a d_{\nabla h_{\tilde{l},+}^{(y)}}^{*}\left(\nabla h_{\tilde{a},+}^{(t)}\right)-a d_{\nabla h_{\tilde{l},+}^{(t)}}^{*}\left(\nabla h_{\tilde{a},+}^{(y)}\right)-\frac{\partial}{\partial t} \nabla h_{\tilde{a},+}^{(y)}+\frac{\partial}{\partial y} \nabla h_{\tilde{a},+}^{(t)}
\end{gathered}
$$

for any $\tilde{a} \ltimes \tilde{l} \in \tilde{\mathcal{G}}$. Thus, the following important proposition holds.

Proposition 2. The Hamiltonian flows (17) generate the separately commuting evolution equations (15) and (16). The evolution equations (15) give rise to the Lax type compatibility condition (19), being equivalent to some system of nonlinear heavenly type equations in partial derivatives.

The presented above construction of Hamiltonian flows on the adjoint space $\tilde{\mathcal{G}}^{*}$ still allows the next important generalization. Namely, let us endow the point product $\tilde{\mathcal{G}}^{S^{1}}:=\prod_{z \in \mathbb{S}^{1}} \tilde{\mathcal{G}}$ of the loop Lie algebra $\tilde{\mathcal{G}}$ with the central extension generated by a two-cocycle $\omega_{2}: \tilde{\mathcal{G}} \times \tilde{\mathcal{G}} \rightarrow \mathbb{C}$, where

$$
\omega_{2}\left(\tilde{a}_{1} \ltimes \tilde{l}_{1}, \tilde{a}_{2} \ltimes \tilde{l}_{2}\right):=\int_{\mathrm{S}^{1}}\left[\left(l_{1}, \partial \tilde{a}_{2} / \partial z\right)-\left(l_{2}, \partial \tilde{a}_{1} / \partial z\right)\right]
$$

for any elements $\tilde{a}_{1} \ltimes \tilde{l}_{1}, \tilde{a}_{2} \ltimes \tilde{l}_{2} \in \tilde{\mathcal{G}}$. The resulting centrally extended Lie-algebra $\tilde{\mathcal{G}}:=\tilde{\mathcal{G}} \oplus \mathbb{C}$ is defined by the commutator

$$
\left[\left(\tilde{a}_{1} \ltimes \tilde{l}_{1} ; \alpha_{1}\right),\left(\tilde{a}_{2} \ltimes \tilde{l}_{2} ; \alpha_{1}\right)\right]:=\left(\left[\tilde{a}_{1}, a_{2}\right] \ltimes\left(a d_{\tilde{a}_{1}}^{*} \tilde{l}_{2}-a d_{\tilde{a}_{2}}^{*} \tilde{l}_{1}\right) ; \omega_{2}\left(\tilde{a}_{1} \ltimes \tilde{l}_{1}, \tilde{a}_{2} \ltimes \tilde{l}_{2}\right)\right.
$$

for any pair of elements $\left(\tilde{a}_{1} \ltimes \tilde{l}_{1} ; \alpha_{1}\right),\left(\tilde{a}_{2} \ltimes \tilde{l}_{2} ; \alpha_{1}\right) \in \tilde{\mathcal{G}}$. The resulting $R$-deformed Lie-Poisson bracket (18) for any smooth functionals $h, f \in D\left(\mathcal{G}^{*}\right)$ on the adjoint space $\tilde{\mathcal{G}}^{*}$ becomes equal to

$$
\begin{aligned}
\{h, f\}_{\mathcal{R}}:=(\tilde{a} \ltimes \tilde{l},[\nabla h(\tilde{l}, \tilde{a}) & \left., \nabla f(\tilde{l}, \tilde{a})]_{\mathcal{R}}\right) \\
& +\omega_{2}(\mathcal{R} \nabla h(\tilde{l}, \tilde{a}), \nabla f(\tilde{l}, \tilde{a}))+\omega_{2}(\nabla h(\tilde{l}, \tilde{a}), \mathcal{R} \nabla f(\tilde{l}, \tilde{a})) .
\end{aligned}
$$

The corresponding Casimir functionals $h^{(p)} \in I\left(\tilde{\mathcal{G}}^{*}\right), p \in \mathbb{Z}_{+}$, are defined with respect to the standard Lie-Poisson bracket as

$$
\left\{h^{(p)}, f\right\}:=\left(\tilde{a} \ltimes \tilde{l},\left[\nabla h^{(p)}(\tilde{l}, \tilde{a}), \nabla f(\tilde{a}, \tilde{l})\right]\right)+\omega_{2}\left(\nabla h^{(p)}(\tilde{a}, \tilde{l}), \nabla f(\tilde{a}, \tilde{l})\right)=0
$$

for all smooth functionals $f \in D\left(\tilde{\mathcal{G}}^{*}\right)$. Based on the equality (21) one easily finds that the gradients $\nabla h^{(p)} \in \tilde{\mathcal{G}}$ of the Casimir functionals $h^{(p)} \in I\left(\tilde{\mathcal{G}}^{*}\right), p \in \mathbb{Z}_{+}$, satisfy the following equations:

$$
\left[\nabla h_{\tilde{l}}, \tilde{a}\right]-\frac{\partial}{\partial z} \nabla h_{\tilde{l}}=0, \quad a d_{\nabla h_{\tilde{l}}}^{*} \tilde{l}-a d_{\tilde{a}}^{*} \nabla h_{\tilde{a}}-\frac{\partial}{\partial z} \nabla h_{\tilde{a}}=0
$$


for any chosen element $\tilde{a} \ltimes \tilde{l} \in \overline{\mathcal{G}}^{*}$. Making use of suitably constructed Casimir functionals $h^{(y)}, h^{(t)} \in I(\tilde{\mathcal{G}})$, one can construct from (20) the following commuting Hamiltonian flows on the adjoint space $\tilde{\mathcal{G}}^{*}$ :

$$
\frac{\partial}{\partial y}(\tilde{a} \ltimes \tilde{l})=\left\{\tilde{a} \ltimes \tilde{l}, h^{(y)}\right\}_{\mathcal{R},} \quad \frac{\partial}{\partial t}(\tilde{a} \ltimes \tilde{l})=\left\{\tilde{a} \ltimes \tilde{l}, h^{(t)}\right\}_{\mathcal{R}},
$$

which are equivalent to the evolution equations

$$
\frac{\partial}{\partial y} \tilde{a}=-\left[\nabla h_{\tilde{l},,^{\prime}}^{(y)} \tilde{a}\right]+\frac{\partial}{\partial z} \nabla h_{\tilde{l},+^{\prime}}^{(y)} \quad \frac{\partial}{\partial t} \tilde{a}=-\left[\nabla h_{\tilde{l},+^{\prime}}^{(t)} \tilde{a}\right]+\frac{\partial}{\partial z} \nabla h_{\tilde{l},+^{\prime}}^{(t)}
$$

and

$$
\begin{gathered}
\frac{\partial}{\partial y} \tilde{l}=-a d_{\nabla h_{\tilde{l},+}^{(y)}}^{*} \tilde{l}+a d_{\tilde{a}}^{*}\left(\nabla h_{\tilde{a}_{,+}}^{(y)}\right)+\frac{\partial}{\partial z} \nabla h_{\tilde{a}_{,+}}^{(y)}, \\
\frac{\partial}{\partial t} \tilde{l}=-a d_{\nabla h_{\tilde{l},+}^{(t)}}^{*} \tilde{l}+a d_{\tilde{a}^{*}}^{*}\left(\nabla h_{\tilde{a}_{,+}}^{(t)}\right)+\frac{\partial}{\partial z} \nabla h_{\tilde{a}_{,+}}^{(t)}
\end{gathered}
$$

The commutativity condition for these flows is split into two equations such as

$$
\left[\nabla h_{\tilde{l},+}^{(y)}, \nabla h_{\tilde{l},+}^{(t)}\right]-\frac{\partial}{\partial t} \nabla h_{\tilde{l},+}^{(y)}+\frac{\partial}{\partial y} \nabla h_{\tilde{l},+}^{(t)}=0,
$$

and

$$
\begin{gathered}
\frac{\partial \tilde{P}}{\partial z}+a d_{\tilde{a}}^{*} \tilde{P}=0, \\
\tilde{P}=a d_{\nabla h_{\tilde{l},+}^{(y)}}^{*}\left(\nabla h_{\tilde{a},+}^{(t)}\right)-a d_{\nabla h_{\tilde{l},+}^{(t)}}^{*}\left(\nabla h_{\tilde{a},+}^{(y)}\right)-\frac{\partial}{\partial t} \nabla h_{\tilde{a},+}^{(y)}+\frac{\partial}{\partial y} \nabla h_{\tilde{a},+}^{(t)}
\end{gathered}
$$

for any $\tilde{a} \ltimes \tilde{l} \in \tilde{\mathcal{G}}$. The first of them can be considered as the Lax type compatibility condition for the evolution equations (23). As a consequence of the obtained above results one can formulate the following proposition.

Proposition 3. The Hamiltonian flows (22) on the adjoint space $\tilde{\mathcal{G}}^{*}$ generate the separately commuting evolution equations (23) and (24). The evolution equations (23) give rise to the Lax type compatibility condition (25), being equivalent to some system of nonlinear heavenly type equations in partial derivatives. Moreover, the system of evolution equations (23) can be considered as the compatibility condition for the following set of linear vector equations

$$
\partial \psi / \partial y+\nabla h_{\tilde{l},+}^{(y)} \psi=0, \quad \partial \psi / \partial z+\tilde{a} \psi=0, \quad \partial \psi / \partial t+\nabla h_{\tilde{l},+}^{(t)} \psi=0
$$

for all $\left.(y, t ; \lambda, z, x) \in \mathbb{R}^{2} \times\left(\mathbb{C} \times \mathbb{S}^{1}\right) \times \mathbb{T}^{n}\right)$ and a function $\psi \in C^{2}\left(\mathbb{R}^{2} \times \mathbb{C} \times\left(\mathbb{S}^{1} \times \mathbb{T}^{n}\right) ; \mathbb{C}\right)$.

The following example demonstrates the analytical applicability of the devised above Liealgebraic scheme for construction a wide class of nonlinear multidimensional heavenly type integrable Hamiltonian systems on functional spaces. 


\subsection{Example: the modified Mikhalev-Pavlov heavenly type system}

Let a seed element $\tilde{a} \ltimes \tilde{l} \in \tilde{\mathcal{G}}^{*}$ be chosen in its reduced form as

$$
\tilde{a} \ltimes \tilde{l}=\left(\left(u_{x}+v_{x} \lambda-\lambda^{2}\right) \partial / \partial x \ltimes\left(w_{x}+\zeta_{x} \lambda\right) d x,\right.
$$

where $u, v, w, \zeta \in \mathbb{C}^{2}\left(\mathbb{R}^{2} \times \mathbb{S}^{1} \times \mathbb{T}^{1} ; \mathbb{R}\right)$. The asymptotic splits for the components of the gradient of the corresponding Casimir functional $h \in I\left(\overline{\mathcal{G}}^{*}\right)$, as $|\lambda| \rightarrow \infty$ have the following forms:

$$
\begin{aligned}
\nabla h_{\tilde{l}} \simeq 1 & -v_{x} \lambda^{-1}-u_{x} \lambda^{-2}-v_{z} \lambda^{-3}-\left(u_{z}+v_{x} v_{z}-2\left(\partial_{x}^{-1} v_{x x} v_{z}\right)\right) \lambda^{-4} \\
& +v_{y} \lambda^{-5}-\left(-u_{y}-v_{x} v_{y}+2\left(\partial_{x}^{-1} v_{x x} v_{y}\right)\right) \lambda^{-6}+\ldots, \\
\nabla h_{\tilde{a}} \simeq & -\zeta_{x} \lambda^{-1}-w_{x} \lambda^{-2}-\zeta_{z} \lambda^{-3}-\left(w_{z}-\zeta_{x} v_{z}+2 v_{x} \zeta_{z}+\left(\partial_{x}^{-1} v_{x} \zeta_{x}\right) z_{z}\right) \lambda^{-4} \\
& +\zeta_{y} \lambda^{-5}-\left(-w_{y}+\zeta_{x} v_{y}-2 v_{x} \zeta_{y}+\left(\partial_{x}^{-1} v_{x} \zeta_{x}\right)_{y}\right) \lambda^{-6}+\ldots
\end{aligned}
$$

In the case when

$$
\begin{aligned}
\nabla h_{\tilde{l},+}^{(y)} & :=\lambda^{4}-v_{x} \lambda^{3}-u_{x} \lambda^{2}-v_{z} \lambda-\left(u_{z}+v_{x} v_{z}-2\left(\partial_{x}^{-1} v_{x x} v_{z}\right)\right), \\
\nabla h_{\tilde{a},+}^{(y)} & :=-\zeta_{x} \lambda^{3}-w_{x} \lambda^{2}-\zeta_{z} \lambda-\left(w_{z}-\zeta_{x} v_{z}+2 v_{x} \zeta_{z}-\left(\partial_{x}^{-1} v_{x} \zeta_{x}\right)_{z}\right),
\end{aligned}
$$

and

$$
\begin{aligned}
\nabla h_{\tilde{l},+}^{(t)}:= & \lambda^{6}-v_{x} \lambda^{5}-u_{x} \lambda^{4}-v_{z} \lambda^{3}-\left(u_{z}+v_{x} v_{z}-2\left(\partial_{x}^{-1} v_{x x} v_{z}\right)\right) \lambda^{2} \\
& +v_{y} \lambda-\left(-u_{y}-v_{x} v_{y}+2\left(\partial_{x}^{-1} v_{x x} v_{y}\right)\right), \\
\nabla h_{\tilde{a},+}^{(t)}:= & -\zeta_{x} \lambda^{5}-w_{x} \lambda^{4}-\zeta_{z} \lambda^{3}-\left(w_{z}-\zeta_{x} v_{z}+2 v_{x} \zeta_{z}-\left(\partial_{x}^{-1} v_{x} \zeta_{x}\right) z\right) \lambda^{2} \\
& +\zeta_{y} \lambda-\left(-w_{y}+\zeta_{x} v_{y}-2 v_{x} \zeta_{y}+\left(\partial_{x}^{-1} v_{x} \zeta_{x}\right)_{y}\right),
\end{aligned}
$$

the compatibility condition of the Hamiltonian vector flows (22) leads to the system of evolution equations:

$$
\begin{aligned}
u_{z t}+u_{y y}= & -u_{y} u_{x z}+u_{z} u_{x y}-v_{y} v_{x y}+v_{z} v_{x t}-u_{z} v_{y} v_{x x}+u_{y} v_{z} v_{x x} \\
& -v_{x}^{2} v_{z} v_{x y}+v_{x}^{2} v_{y} v_{x z}-2 e u_{x y}-2 s u_{x z}+2 e_{t}-2 s_{y}+2 e v_{y} v_{x x}+2 s v_{z} v_{x x}, \\
v_{z t}+v_{y y}= & -u_{y} v_{x z}+u_{z} v_{x y}-v_{y} u_{x z}+v_{z} u_{x y}-2 e v_{x y}-2 s v_{x z}-2 v_{x} v_{y} v_{x z}+2 v_{x} v_{z} v_{x y}, \\
-u_{x y}-u_{z z}= & u_{x} u_{x z}-u_{z} u_{x x}-u_{x x} v_{x} v_{z}+u_{x} v_{x z} v_{x}-u_{x} v_{x x} v_{z}+\left(v_{x} v_{z}\right)_{z}+2 u_{x x} e-2 e_{z}, \\
-v_{x y}-v_{z z}= & u_{x z} v_{x}-u_{z} v_{x x}-u_{x x} v_{z}+u_{x} v_{x z}-2 v_{x x} v_{x} v_{z}+v_{x}^{2} v_{x z}+2 v_{x x} e \\
-u_{x t}+u_{y z}= & -u_{x} u_{x y}+u_{y} u_{x x}+u_{x x} v_{x} v_{y}-u_{x} v_{x y} v_{x}+u_{x} v_{x x} v_{y}-\left(v_{x} v_{y}\right)_{z}+2 u_{x x} s-2 s_{z}, \\
-v_{x t}+v_{y z}= & -u_{x y} v_{x}+u_{y} v_{x x}+u_{x x} v_{y}-u_{x} v_{x y}+2 v_{x x} v_{x} v_{y}-v_{x}^{2} v_{x y}+2 v_{x x} s,
\end{aligned}
$$

where

$$
e_{x x}=v_{x x} v_{z}, \quad s_{x x}=-v_{x x} v_{y} .
$$

Under the constraint $v=0$ one obtains a set of independent scalar differential equations before listed in $[17,18,23]$; two equations are spatially four-dimensional:

$$
u_{z t}+u_{y y}=-u_{y} u_{x z}+u_{z} u_{x y}
$$

and

$$
-u_{x t}+u_{y z}=-u_{x} u_{x y}+u_{y} u_{x x},
$$


a one is spatially three-dimensional:

$$
-u_{x y}-u_{z z}=u_{x} u_{x z}-u_{z} u_{x x}
$$

In particular, under the spatial variable reductions $x \rightarrow y \in \mathbb{R}, t \rightarrow z \in \mathbb{R}$, the second equation becomes trivial and the first (32) and third (31) equations bring about the reduced MikhalevPavlov type equation

$$
u_{z z}+u_{y y}=-u_{y} u_{y z}+u_{z} u_{y y}
$$

Proposition 4. The constructed set of heavenly type equations (27), (28) has the Lax-Sato vector field representation (19) with the "spectral" parameter $\lambda \in \mathbb{C}$, which is related with the seed element $\tilde{a} \ltimes \tilde{l} \in \tilde{\mathcal{G}}^{*}$ in the form (26).

Remark 1. The following remark concerning the dimensionality of the differential systems obtained above proves to be essential. The generalized Mikhalev-Pavlov differential system (29) as the one considered on the related jet-manifold $J\left(\mathbb{R}^{4} ; \mathbb{R}^{2}\right)$ for smooth mappings $(u, v)$ : $\mathbb{R}^{4} \rightarrow \mathbb{R}^{2}$ presents, in reality, a differential system with effective dimension equal $2=4-2$. This fact is important from the geometric point of view devised recently in E. V. Ferapontov and others [19,22] works, devoted to the Plücker manifold imbedding into the Grassmannians and a classification of related integrable differential systems. There was, in particular, stated that the corresponding integrable systems associated with fourfolds in $G r(3,5)$ also appeared to be effectively two-dimensional, ensuing at the present time in some sense a challenging problem. As it was also mentioned above concerning a generalization of spatially multidimensional Mikhalev-Pavlov type equations by means of the seed element (33), there is a possibility to check directly the existence of effectively three and more dimensional integrable differential systems and then, eventually, to construct them.

We can here observe that the seed element (26) can be presented in the following special compact form:

$$
\tilde{a} \ltimes \tilde{l}:=\frac{d \tilde{\eta}}{d x} \partial / \partial x \ltimes d \tilde{\rho}, \tilde{\eta}=u+v \lambda-\lambda^{2} x, \tilde{\rho}=w+\zeta \lambda,
$$

deeply connected with geometry of the related moduli space of flat connections, related to coadjoint actions of the corresponding Casimir functionals. Its possible generalization to spatially multidimensional Mikhalev-Pavlov type equations can be done by the seed element

$$
\tilde{a} \ltimes \tilde{l}:=\langle\nabla \tilde{\eta}, \nabla\rangle \ltimes d \tilde{\rho}
$$

for some elements $\tilde{\eta}, \tilde{\rho} \in \Omega^{0}\left(\mathbb{T}^{n}\right) \otimes \mathbb{C}, n \in \mathbb{N}$. An analysis of the case (33) and corresponding systems of spatially multidimensional Mikhalev-Pavlov type equations is planned to be done in a separate study.

\subsection{The modified Martinez Alonso-Shabat heavenly type system}

If the seed element $\tilde{a} \ltimes \tilde{l} \in \tilde{\mathcal{G}}^{*}$ is chosen in its reduced form as

$$
\begin{aligned}
\tilde{a} \ltimes \tilde{l} & =\left(\left(\left(u_{x_{1}}+c u_{x_{2}}\right)+\lambda\right) \partial / \partial x_{1}+\left(\left(v_{x_{1}}+c v_{x_{2}}\right)+c \lambda\right) \partial / \partial x_{2}\right) \\
& \ltimes\left(\left(w_{x_{1}}+c w_{x_{2}}\right) d x_{1}+\left(\zeta_{x_{1}}+c \zeta_{x_{2}}\right) d x_{2}\right),
\end{aligned}
$$


where $u, v, w, \zeta \in C^{2}\left(\mathbb{R}^{2} \times \mathbb{S}^{1} \times \mathbb{T}^{2} ; \mathbb{R}\right), c \in \mathbb{R} \backslash\{0\}$, one has the following asymptotic splits for the components of the gradients of the corresponding Casimir functionals $h^{(1)}, h^{(2)} \in I\left(\overline{\mathcal{G}}^{*}\right)$ as $|\lambda| \rightarrow \infty:$

$$
\begin{aligned}
& \nabla h_{\tilde{l}}^{(1)} \simeq\left(\begin{array}{c}
1+\left(u_{x_{1}}+c u_{x_{2}}\right) \lambda^{-1}-u_{z} \lambda^{-2}+\ldots \\
c+\left(v_{x_{1}}+c v_{x_{2}}\right) \lambda^{-1}-v_{z} \lambda^{-2}+\ldots
\end{array}\right), \\
& \nabla h_{\tilde{a}}^{(1)} \simeq\left(\begin{array}{c}
\left(w_{x_{1}}+c w_{x_{2}}\right) \lambda^{-1}-w_{z} \lambda^{-2}+\ldots \\
\left(\zeta_{x_{1}}+c \zeta_{x_{2}}\right) \lambda^{-1}-\zeta_{z} \lambda^{-2}+\ldots
\end{array}\right),
\end{aligned}
$$

and

$$
\begin{aligned}
& \nabla h_{\tilde{l}}^{(2)} \simeq\left(\begin{array}{c}
1+\left(u_{x_{1}}-c u_{x_{2}}\right) \lambda^{-1}+\varkappa \lambda^{-2}+\ldots \\
-c+\left(v_{x_{1}}-c v_{x_{2}}\right) \lambda^{-1}+\omega \lambda^{-2}+\ldots
\end{array}\right), \\
& \nabla h_{\tilde{a}}^{(2)} \simeq\left(\begin{array}{c}
\left(w_{x_{1}}-c w_{x_{2}}\right) \lambda^{-1}+\varrho \lambda^{-2}+\ldots \\
\left(\zeta_{x_{1}}-c \zeta_{x_{2}}\right) \lambda^{-1}+\chi \lambda^{-2}+\ldots
\end{array}\right),
\end{aligned}
$$

where

$$
\begin{aligned}
& \varkappa_{x_{1}}+c \varkappa_{x_{2}}=-\left(u_{z x_{1}}-c u_{z x_{2}}\right)+2 c\left(u_{x_{1}} u_{x_{1} x_{2}}-u_{x_{2}} u_{x_{1} x_{1}}+v_{x_{1}} u_{x_{2} x_{2}}-v_{x_{2}} u_{x_{1} x_{2}}\right) \\
& \omega_{x_{1}}+c \omega_{x_{2}}=-\left(v_{z x_{1}}-c v_{z x_{2}}\right)+2 c\left(u_{x_{1}} v_{x_{1} x_{2}}-u_{x_{2}} v_{x_{1} x_{1}}+v_{x_{1}} v_{x_{2} x_{2}}-v_{x_{2}} v_{x_{1} x_{2}}\right)
\end{aligned}
$$

and

$$
\begin{aligned}
\varrho_{x_{1}}+c \varrho_{x_{2}}= & -\left(w_{z x_{1}}-c w_{z x_{2}}\right)+2 c\left(u_{x_{1}} w_{x_{1} x_{2}}-u_{x_{2}} w_{x_{1} x_{1}}+2 w_{x_{2}} u_{x_{1} x_{1}}\right. \\
& \left.-2 w_{x_{1}} u_{x_{1} x_{2}}+v_{x_{1}} w_{x_{2} x_{2}}-v_{x_{2}} w_{x_{1} x_{2}}+w_{x_{2}} v_{x_{1} x_{2}}-w_{x_{2}} v_{x_{2} x_{2}}+\zeta_{x_{2}} v_{x_{1} x_{1}}-\zeta_{x_{1}} v_{x_{1} x_{2}}\right), \\
\chi_{x_{1}}+c \chi_{x_{2}}= & -\left(\zeta_{z x_{1}}-c \zeta_{z x_{2}}\right)+2 c\left(v_{x_{1}} \zeta_{x_{2} x_{2}}-v_{x_{2}} \zeta_{x_{1} x_{2}}+2 \zeta_{x_{2}} v_{x_{1} x_{2}}\right. \\
& \left.-2 \zeta_{x_{1}} v_{x_{2} x_{2}}+u_{x_{1}} \zeta_{x_{1} x_{2}}-u_{x_{2}} \zeta_{x_{1} x_{1}}+\zeta_{x_{2}} u_{x_{1} x_{1}}-\zeta_{x_{1}} u_{x_{1} x_{2}}+w_{x_{2}} u_{x_{1} x_{2}}-w_{x_{1}} u_{x_{2} x_{2}}\right) .
\end{aligned}
$$

In the case when

$$
\begin{aligned}
& \nabla h_{\tilde{l},+}^{(y)}:=\left(\begin{array}{c}
\lambda^{2}+\left(u_{x_{1}}+c u_{x_{2}}\right) \lambda-u_{z} \\
c \lambda^{2}+\left(v_{x_{1}}+c v_{x_{2}}\right) \lambda-v_{z}
\end{array}\right), \\
& \nabla h_{\tilde{a},+}^{(y)}:=\left(\begin{array}{c}
\left(w_{x_{1}}+c w_{x_{2}}\right) \lambda-w_{z} \\
\left(\zeta_{x_{1}}+c \zeta_{x_{2}}\right) \lambda-\zeta_{z}
\end{array}\right),
\end{aligned}
$$

and

$$
\begin{aligned}
& \nabla h_{\tilde{l},+}^{(t)}:=\left(\begin{array}{c}
\lambda^{2}+\left(u_{x_{1}}-c u_{x_{2}}\right) \lambda+\varkappa \\
-c \lambda^{2}+\left(v_{x_{1}}-c v_{x_{2}}\right) \lambda+\omega
\end{array}\right), \\
& \nabla h_{\tilde{a},+}^{(t)}:=\left(\begin{array}{c}
\left(w_{x_{1}}-c w_{x_{2}}\right) \lambda+\varrho \\
\left(\zeta_{x_{1}}-c \zeta_{x_{2}}\right) \lambda+\chi
\end{array}\right),
\end{aligned}
$$


the compatibility condition of the Hamiltonian vector flows (22) leads to the system of evolution equations:

$$
\begin{aligned}
u_{z t}+\varkappa_{y}= & -u_{z x_{1}} \varkappa-u_{z x_{2}} \omega+u_{z} \varkappa_{x_{1}}+v_{z} \varkappa_{x_{2}} \\
v_{z t}+\omega_{y}= & -v_{z x_{1}} \varkappa-v_{z x_{2}} \omega+u_{z} \omega_{x_{1}}+v_{z} \omega_{x_{2}}, \\
u_{y x_{1}}+c u_{y x_{2}}= & -\left(u_{x_{1}}+c u_{x_{2}}\right) u_{z x_{1}}-\left(v_{x_{1}}+c v_{x_{2}}\right) u_{z x_{2}}+\left(u_{x_{1} x_{1}}+c u_{x_{1} x_{2}}\right) u_{z} \\
& +\left(u_{x_{1} x_{2}}+c u_{x_{2} x_{2}}\right) v_{z}-u_{z z}, \\
v_{y x_{1}}+c v_{y x_{2}}= & -\left(u_{x_{1}}+c u_{x_{2}}\right) v_{z x_{1}}-\left(v_{x_{1}}+c v_{x_{2}}\right) v_{z x_{2}}+\left(v_{x_{1} x_{1}}+c v_{x_{1} x_{2}}\right) u_{z} \\
& +\left(v_{x_{1} x_{2}}+c v_{x_{2} x_{2}}\right) v_{z}-v_{z z} \\
u_{t x_{1}}+c u_{t x_{2}}= & \left(u_{x_{1}}+c u_{x_{2}}\right) \varkappa_{x_{1}}+\left(v_{x_{1}}+c v_{x_{2}}\right) \varkappa_{x_{2}}-\left(u_{x_{1} x_{1}}+c u_{x_{1} x_{2}}\right) \varkappa \\
& -\left(u_{x_{1} x_{2}}+c u_{x_{2} x_{2}}\right) \omega+\varkappa_{z} \\
v_{t x_{1}}+c v_{t x_{2}}= & \left(u_{x_{1}}+c u_{x_{2}}\right) \omega_{x_{1}}+\left(v_{x_{1}}+c v_{x_{2}}\right) \omega_{x_{2}}-\left(v_{x_{1} x_{1}}+c v_{x_{1} x_{2}}\right) \varkappa \\
& -\left(v_{x_{1} x_{2}}+c v_{x_{2} x_{2}}\right) \omega+\omega_{z} .
\end{aligned}
$$

Thus, the following proposition holds.

Proposition 5. The constructed system of heavenly type equations (36) and (35) has the LaxSato vector field representation (19) with the "spectral" parameter $\lambda \in \mathbb{C}$, which is related with the element $\tilde{a} \ltimes \tilde{l} \in \tilde{\mathcal{G}}^{*}$ in the form (34).

The system of equations (36) and (35) admits the reduction when $v=u$ and $\omega=\varkappa$. In this case, under $c=1$ one obtains

$$
\begin{aligned}
u_{z t}+\varkappa_{y} & =-\left(u_{z x_{1}}+u_{z x_{2}}\right) \varkappa+u_{z}\left(\varkappa_{x_{1}}+\varkappa_{x_{2}}\right), \\
\varkappa_{x_{1}}+\varkappa_{x_{2}} & \left.=-\left(u_{z x_{1}}-u_{z x_{2}}\right)-2\left(\left(u_{x_{1}} u_{x_{2}}\right)\right)_{x_{1}}-\left(u_{x_{1}} u_{x_{2}}\right) x_{2}\right) .
\end{aligned}
$$

The change $u_{z}=u_{x_{1}}+u_{x_{2}}$ in (37) leads to the system:

$$
\begin{aligned}
\left(u_{\tilde{t} x_{1}}+u_{\tilde{t} x_{2}}\right)-\left(u_{\tilde{y} x_{1}}-u_{\tilde{y} x_{2}}\right) & =u_{x_{1} x_{2}}\left(u_{x_{1}}-u_{x_{2}}\right)-u_{x_{1} x_{1}} u_{x_{2}}+u_{x_{2} x_{2}} u_{x_{1}} \\
& -u_{x_{1} x_{2}}\left(u_{x_{1}}^{2}-u_{x_{2}}^{2}\right)-u_{x_{1} x_{1}} u_{x_{2}}\left(u_{x_{1}}+u_{x_{2}}\right)+u_{x_{2} x_{2}} u_{x_{1}}\left(u_{x_{1}}+u_{x_{2}}\right) \\
& -2 \rho_{\tilde{y}}+\left(u_{x_{1} x_{1}}+2 u_{x_{1} x_{2}}+u_{x_{2} x_{2}}\right) \rho \\
\rho_{x_{1}}+\rho_{x_{2}} & \left.=\left(u_{x_{1}} u_{x_{2}}\right)_{x_{1}}-\left(u_{x_{1}} u_{x_{2}}\right)\right)_{x_{2}}
\end{aligned}
$$

where $\tilde{t}=2 t$ and $\tilde{y}=2 y$. Thus, the system (37) can be considered as some modification of the Martinez Alonso-Shabat one [3].

\section{HEAVENLY TYPE SYSTEMS: THE GENERALIZED LIE-ALGEBRAIC STRUCTURES}

Concerning a further generalization of the multi-dimensional case related with the loop group $\widetilde{\operatorname{Diff}}\left(\mathbb{T}^{n}\right)$ on the torus $\mathbb{T}^{n}, n \in \mathbb{Z}_{+}$, one can proceed, as before, [25] the following natural way: as the Lie algebra $\widetilde{\operatorname{dif} f}\left(\mathbb{T}^{n}\right)$ consists of the loop group elements, holomorphically continued from the circle $S^{1}:=\partial \mathbb{D}^{1}$, being the boundary of the disk $\mathbb{D}^{1} \subset \mathbb{C}$, by means of the complex "spectral" variable $\lambda \in \mathbb{C}$ both into the interior $\mathbb{D}_{+}^{1} \subset \mathbb{C}$ and the exterior $\mathbb{D}_{-}^{1} \subset \mathbb{C}$ parts of the disk $\mathbb{D}^{1} \subset \mathbb{C}$, one can take into account its analytical invariance subject to the 
circle $\mathrm{S}^{1}:=\partial \mathbb{D}^{1}$ diffeomorphism group $\operatorname{Diff}\left(\mathrm{S}^{1}\right)$. The latter gives rise to the naturally extended holomorphic Lie algebra $\left.\widetilde{\operatorname{diff}}\left(\mathbb{T}^{n}\right)=\widetilde{\operatorname{diff}}+\mathbb{T}^{n}\right)_{+} \oplus \widetilde{\operatorname{diff} f_{-}}\left(\mathbb{T}^{n}\right)$ on the Cartesian product $\mathbb{C} \times \mathbb{T}^{n}$, whose elements are representable as

$$
\bar{a}:=\left\langle a(x ; \lambda), \frac{\partial}{\partial x}\right\rangle=a_{0}(x ; \lambda) \frac{\partial}{\partial \lambda}+\sum_{j=1}^{n} a_{j}(x ; \lambda) \frac{\partial}{\partial x_{j}}
$$

for some holomorphic in $\lambda \in \mathbb{D}_{ \pm}^{1}$ vectors $a(x ; \lambda) \in E \times E^{n}$ for all $x \in \mathbb{T}^{n}$, and where we denoted by $\frac{\partial}{\partial x}:=\left(\frac{\partial}{\partial \lambda}, \frac{\partial}{\partial x_{1}}, \frac{\partial}{\partial x_{2}}, \ldots, \frac{\partial}{\partial x_{n}}\right)^{\top}$ the generalized Euclidean vector gradient with respect to the vector variable $x:=(\lambda, x) \in \mathbb{T}^{n}$.

Construct now the semi-direct sum $\overline{\mathcal{G}}:=\operatorname{diff}\left(\mathbb{T}^{n}\right) \ltimes \operatorname{diff}\left(\mathbb{T}^{n}\right)^{*}$ of the loop Lie algebra $\operatorname{diff}\left(\mathbb{T}^{n}\right)$ and its adjoint space $\operatorname{diff}\left(\mathbb{T}^{n}\right)^{*}$, taking into account their natural pairing

$$
(\bar{l} \mid \bar{a}):=\underset{\lambda \in \mathbb{C}}{\operatorname{res}}(l(\mathbf{x}) \mid a(\mathbf{x}))_{H^{0}}
$$

for any $\bar{l}:=\langle l(x ; \lambda), d x\rangle=l_{0}(x ; \lambda) d \lambda+\sum_{j=1}^{n} l_{j}(x ; \lambda) d x_{j} \in \operatorname{diff}\left(\mathbb{T}^{n}\right)^{*}$ and $\bar{a} \in \operatorname{diff}\left(\mathbb{T}^{n}\right)$. The corresponding Lie commutator on the loop Lie algebra $\overline{\mathcal{G}}$ is naturally given by the expression

$$
\left[\bar{a}_{1} \ltimes \bar{l}_{1}, \bar{a}_{2} \ltimes \bar{l}_{2}\right]=\left[\bar{a}_{1}, a_{2}\right] \ltimes a d_{a_{2}}^{*} \bar{l}_{1}-a d_{a_{1}}^{*} \bar{l}_{2}
$$

for any $\bar{a}_{1} \ltimes \bar{l}_{1}, \bar{a}_{2} \ltimes \bar{l}_{2} \in \overline{\mathcal{G}}$. The Lie algebra $\overline{\mathcal{G}}$ also splits into the direct sum of two subalgebras

$$
\overline{\mathcal{G}}=\overline{\mathcal{G}}_{+} \oplus \overline{\mathcal{G}}_{-},
$$

allowing to introduce on it the classical $R$-structure

$$
\left[\bar{a}_{1} \ltimes \bar{l}_{1}, \bar{a}_{2} \ltimes \bar{l}_{2}\right]_{\mathcal{R}}:=\left[\mathcal{R}\left(\bar{a}_{1} \ltimes \bar{l}_{1}\right), \bar{a}_{2} \ltimes \bar{l}_{2}\right]+\left[\bar{a}_{1} \ltimes \bar{l}_{1}, \mathcal{R}\left(\bar{a}_{2} \ltimes \bar{l}_{2}\right)\right]
$$

for any $\bar{a}_{1} \ltimes \bar{l}_{1}, \bar{a}_{2} \ltimes \bar{l}_{2} \in \overline{\mathcal{G}}$, where, by definition,

$$
\mathcal{R}:=\left(P_{+}-P_{-}\right) / 2, \quad \text { and } \quad P_{ \pm} \overline{\mathcal{G}}:=\overline{\mathcal{G}}_{ \pm} \subset \overline{\mathcal{G}} .
$$

The space $\overline{\mathcal{G}}^{*}$ adjoint to the Lie algebra $\overline{\mathcal{G}}$ can be functionally identified with the space $\overline{\mathcal{G}}$ subject to the nondegenerate symmetric product

$$
(\bar{a} \ltimes \bar{l} \mid \bar{r} \ltimes \bar{m}):=\underset{\lambda \in \mathbb{C}}{\operatorname{res}}(\bar{a} \ltimes \bar{l} \mid \bar{r} \ltimes \bar{m})_{H^{0}},
$$

where we put, by definition, that

$$
(\bar{a} \ltimes \bar{l} \mid \bar{r} \ltimes \bar{m})_{H^{0}}=(\bar{m} \mid \bar{a})_{H^{0}}+(\bar{l} \mid \bar{r})_{H^{0}}
$$

for any pair of elements $\bar{a} \ltimes \bar{l}, \bar{r} \ltimes \bar{m} \in \overline{\mathcal{G}}$.

Owing to the convolution (38), the Lie algebra $\overline{\mathcal{G}}$ becomes metricized. If now to take arbitrary smooth functions $f, g \in D\left(\overline{\mathcal{G}}^{*}\right)$, one can naturally determine two Lie-Poisson brackets

$$
\{f, g\}:=(\bar{a} \ltimes \bar{l} \mid[\nabla f(\bar{l}, \bar{a}), \nabla g(\bar{l}, \bar{a})])
$$

and

$$
\{f, g\}_{\mathcal{R}}:=\left(\bar{a} \ltimes \bar{l} \mid[\nabla f(\bar{l}, \bar{a}), \nabla g(\bar{l}, \bar{a})]_{\mathcal{R}}\right),
$$


where at any seed element $\bar{a} \ltimes \bar{l} \in \overline{\mathcal{G}}^{*} \simeq \overline{\mathcal{G}}$ the gradient element $\nabla f(\bar{l}, \bar{a}):=\nabla f_{\bar{l}} \ltimes \nabla f_{\bar{a}} \simeq$ $\left\langle\nabla f(l, a),(\partial / \partial x, d x)^{\top}\right\rangle \in \overline{\mathcal{G}}$ and $\nabla f_{\bar{l}}=\left\langle\nabla f_{l}, \partial / \partial x\right\rangle, \nabla f_{\bar{a}}=\left\langle\nabla f_{a}, d x\right\rangle$, and, similarly, the gradient element $\nabla g(\bar{l}, \bar{a}):=\nabla g_{\bar{l}} \ltimes \nabla g_{\bar{a}} \simeq\left\langle\nabla g(l, a),(\partial / \partial x, d x)^{\top}\right\rangle \in \overline{\mathcal{G}}^{*}$ and $\nabla g_{\bar{l}}=\left\langle\nabla g_{l}, \partial / \partial x\right\rangle$, $\nabla g_{\bar{a}}=\left\langle\nabla g_{a}, d x\right\rangle$ are calculated with respect to the metric (38).

Let now assume that a smooth function $h \in I\left(\overline{\mathcal{G}}^{*}\right)$ is a Casimir invariant, that is

$$
a d_{\nabla h(\bar{l}, \bar{a})}^{*}(\bar{a} \ltimes \bar{l})=0
$$

for a chosen seed element $\bar{a} \ltimes \bar{l} \in \overline{\mathcal{G}}^{*} \simeq \overline{\mathcal{G}}$. Since for an element $\bar{a} \ltimes \bar{l} \in \overline{\mathcal{G}}^{*} \simeq \overline{\mathcal{G}}$ and arbitrary $f \in D\left(\overline{\mathcal{G}}^{*}\right)$ the adjoint mapping

$$
a d_{\nabla f(\bar{l}, \bar{a})}^{*}(\bar{a} \ltimes \bar{l})=\left(\left[\nabla h_{\bar{l}}, \bar{a}\right] \ltimes\left(a d_{\nabla h_{\bar{l}}}^{*} \bar{l}-a d_{\bar{a}}^{*} \nabla h_{\bar{a}}\right),\right.
$$

the condition (40) can be rewritten as

$$
\left[\nabla h_{\bar{l}}, \bar{a}\right]=0, \quad a d_{\nabla h_{\bar{l}}}^{*} \bar{l}-a d_{\bar{a}}^{*} \nabla h_{\bar{a}}=0,
$$

from which one easily obtains that the Casimir functional $h \in I\left(\overline{\mathcal{G}}^{*}\right)$ satisfies the system of determining equations

$$
\begin{aligned}
& \left\langle\nabla h_{l}, \partial / \partial \mathrm{x}\right\rangle a-\langle a, \partial / \partial \mathrm{x}\rangle \nabla h_{l}=0, \\
& \left\langle\partial / \partial \mathrm{x}, \nabla h_{l}\right\rangle l+\left\langle l,\left(\partial / \partial \mathrm{x} \nabla h_{l}\right)\right\rangle-\langle\partial / \partial \mathrm{x}, a\rangle \nabla h_{a}-\left\langle a,\left(\partial / \partial \mathrm{x} \nabla h_{a}\right)\right\rangle=0 .
\end{aligned}
$$

For the Casimir functional $h \in D\left(\overline{\mathcal{G}}^{*}\right)$ the equations (41) should be solved analytically. In the case when an element $\bar{l} \ltimes \bar{a} \in \overline{\mathcal{G}}^{*}$ is singular as $|\lambda| \rightarrow \infty$, one can consider the general asymptotic expansion

$$
\nabla h^{(p)}(l, a) \sim \lambda^{p} \sum_{j \in \mathbb{Z}_{+}}\left(\nabla h_{l, j}^{(p)} ; \nabla h_{a, j}^{(p)}\right) \lambda^{-j}
$$

for some suitably chosen $p \in \mathbb{Z}_{+}$, which is substituted into the equations (41). The latter is then solved recurrently giving rise to a set of gradient expressions for the Casimir functionals $h^{(p)} \in D\left(\overline{\mathcal{G}}^{*}\right)$ at the specially found integers $p \in \mathbb{Z}_{+}$.

Assume now that $h^{(y)}, h^{(t)} \in I\left(\overline{\mathcal{G}}^{*}\right)$ are such Casimir functionals for which the Hamiltonian vector field generators

$$
\nabla h^{(y)}(\bar{l}, \bar{a})_{+}:=\left(\nabla h^{\left(p_{y}\right)}(\bar{l}, \bar{a})\right)_{+}, \quad \nabla h^{(t)}(\bar{l}, \bar{a})_{+}:=\left(\nabla h^{\left(p_{t}\right)}(\bar{l}, \bar{a})\right)_{+},
$$

where $\nabla h^{(y)}(\bar{l}, \bar{a})_{+}:=\left(\nabla h_{\bar{l}_{,+}}^{(y)} \ltimes \nabla h_{\bar{a}_{,+}}^{(y)}\right) \in \overline{\mathcal{G}}_{+}$and $\nabla h^{(t)}(\bar{l}, \bar{a})_{+}:=\left(\nabla h_{\bar{l}_{,+}}^{(t)} \ltimes \nabla h_{\bar{a}_{1_{+}}}^{(t)}\right) \in \overline{\mathcal{G}}_{+}$, are, respectively, defined at some specially found integers $p_{y}, p_{t} \in \mathbb{Z}_{+}$. These invariants generate owing to the Lie-Poisson bracket (39) the following commuting to each other Hamiltonian flows:

$$
\begin{aligned}
\frac{\partial}{\partial y}(\bar{a} \ltimes \bar{l}) & =-a d_{\nabla h}^{*(y)(\bar{l}, \bar{a})_{+}}(\bar{a} \ltimes \bar{l}), \\
\frac{\partial}{\partial t}(\bar{a} \ltimes \bar{l}) & =-a d_{\nabla h^{(t)}(\bar{l}, \bar{a})_{+}}^{*}(\bar{a} \ltimes \bar{l})
\end{aligned}
$$

of an element $\bar{a} \ltimes \bar{l} \in \overline{\mathcal{G}}^{*} \simeq \overline{\mathcal{G}}$ with respect to the corresponding evolution parameters $t, y \in R$. The flows (43) can be rewritten as

$$
\begin{gathered}
\partial a / \partial y=-\left\langle\nabla h_{l}^{\left(p_{y}\right)}, \frac{\partial}{\partial \mathrm{x}}\right\rangle a+\left\langle a, \frac{\partial}{\partial \mathrm{x}}\right\rangle \nabla h_{l}^{\left(p_{y}\right)}, \\
\partial a / \partial t=-\left\langle\nabla h_{l}^{\left(p_{t}\right)}, \frac{\partial}{\partial \mathrm{x}}\right\rangle a+\left\langle a, \frac{\partial}{\partial \mathrm{x}}\right\rangle \nabla h_{l}^{\left(p_{t}\right)},
\end{gathered}
$$


and

$$
\begin{aligned}
& \partial l / \partial y=-\left\langle\frac{\partial}{\partial \mathrm{x}}, \nabla h_{l}^{\left(p_{y}\right)}\right\rangle l-\left\langle l,\left(\frac{\partial}{\partial \mathrm{x}} \nabla h_{l}^{\left(p_{y}\right)}\right)\right\rangle+\left\langle\frac{\partial}{\partial \mathrm{x}}, a\right\rangle \nabla h_{a}^{\left(p_{y}\right)}+\left\langle a,\left(\frac{\partial}{\partial \mathrm{x}} \nabla h_{a}^{\left(p_{y}\right)}\right)\right\rangle, \\
& \partial l / \partial t=-\left\langle\frac{\partial}{\partial \mathrm{x}}, \nabla h_{l}^{\left(p_{t}\right)}\right\rangle l-\left\langle l,\left(\frac{\partial}{\partial \mathrm{x}} \nabla h_{l}^{\left(p_{t}\right)}\right)\right\rangle+\left\langle\frac{\partial}{\partial \mathrm{x}}, a\right\rangle \nabla h_{a}^{\left(p_{t}\right)}+\left\langle a,\left(\frac{\partial}{\partial \mathrm{x}} \nabla h_{a}^{\left(p_{t}\right)}\right)\right\rangle,
\end{aligned}
$$

where $y, t \in \mathbb{R}$ are the corresponding evolution parameters. Since the invariants $h^{(y)}, h^{(t)} \in$ $I\left(\overline{\mathcal{G}}^{*}\right)$ are commuting to each other with respect to the Lie-Poisson bracket (39), the flows (44) are commuting too. This is equivalent that the following equalities

$$
\left[\nabla h_{\bar{l},+}^{(y)}, \nabla h_{\bar{l},+}^{(t)}\right]-\frac{\partial}{\partial t} \nabla h_{\bar{l},+}^{(y)}+\frac{\partial}{\partial y} \nabla h_{\bar{l},+}^{(t)}=0,
$$

and

$$
\begin{gathered}
a d_{\bar{a}}^{*} \bar{P}=0, \\
\bar{P}=a d_{\nabla h_{\bar{l},+}^{(y)}}^{*}\left(\nabla h_{\bar{a},+}^{(t)}\right)-a d_{\nabla h_{\bar{l},+}^{(t)}}^{*}\left(\nabla h_{\bar{a},+}^{(y)}\right)-\frac{\partial}{\partial t} \nabla h_{\bar{a},+}^{(y)}+\frac{\partial}{\partial y} \nabla h_{\bar{a},+}^{(t)}
\end{gathered}
$$

hold for any $\bar{a} \ltimes \bar{l} \in \overline{\mathcal{G}}$. On the other hand, the equation (45) is equivalent to the compatibility condition of three linear equations

$$
\frac{\partial \psi}{\partial y}+\nabla h_{\bar{l},+}^{(y)} \psi=0, \quad\langle a, \partial / \partial \mathrm{x}\rangle \psi=0, \quad \frac{\partial \psi}{\partial t}+\nabla h_{\bar{l},+}^{(t)} \psi=0
$$

for a function $\psi \in C^{2}\left(\mathbb{R}^{2} \times \mathbb{C} \times \mathbb{T}^{n} ; \mathbb{C}\right)$, all $y, t \in \mathbb{R}$ and any $x \in \mathbb{T}^{n}$. The obtained above results can be formulated as the following proposition.

Proposition 6. Let a seed element $\bar{a} \ltimes \bar{l} \in \overline{\mathcal{G}}^{*}$ and $h^{(y)}, h^{(t)} \in I\left(\overline{\mathcal{G}}^{*}\right)$ are some Casimir functionals subject to the product $(\cdot \mid \cdot)$ on the holomorphic Lie algebra $\overline{\mathcal{G}}$ and the natural coadjoint action on the co-algebra $\overline{\mathcal{G}}^{*} \simeq \overline{\mathcal{G}}$. Then the following dynamical systems

$$
\frac{\partial}{\partial y}(\bar{a} \ltimes \bar{l})=-a d_{\nabla h^{(y)}(\bar{l}, \bar{a})_{+}}(\bar{a} \ltimes \bar{l}), \quad \frac{\partial}{\partial t}(\bar{a} \ltimes \bar{l})=-a d_{\nabla h^{(t)}(\bar{l}, \bar{a})_{+}}(\bar{a} \ltimes \bar{l})
$$

are commuting to each other Hamiltonian flows for evolution parameters $y, t \in R$. Moreover, the compatibility condition of these flows leads to the vector field representation (46).

Remark 2. As it was mentioned above, the expansion (42) is effective if a chosen seed element $\bar{a} \ltimes \bar{l} \in \overline{\mathcal{G}}^{*}$ is singular as $|\lambda| \rightarrow \infty$. In the case when it is singular as $|\lambda| \rightarrow 0$, the expression (42) should be respectively replaced by the expansion

$$
\nabla h^{(p)}(\bar{l}, \bar{a}) \sim \lambda^{-p} \sum_{j \in \mathbb{Z}_{+}} \nabla h_{j}^{(p)}(\bar{l}, \bar{a}) \lambda^{j}
$$

for suitably chosen integers $p \in \mathbb{Z}_{+}$, and the reduced Casimir function gradients then are given by the Hamiltonian vector field generators

$$
\nabla h^{(y)}(\bar{l}, \bar{a})_{-}:=\lambda\left(\lambda^{-p_{y}-1} \nabla h^{\left(p_{y}\right)}(\bar{l}, \bar{a})\right)_{-}, \quad \nabla h^{(t)}(\bar{l}, \bar{a})_{-}:=\lambda\left(\lambda^{-p_{t}-1} \nabla h^{\left(p_{t}\right)}(\bar{l}, \bar{a})\right)_{-}
$$

for suitably chosen positive integers $p_{y}, p_{t} \in \mathbb{Z}_{+}$and the corresponding Hamiltonian flows are, respectively, written as

$$
\frac{\partial}{\partial t}(\bar{a} \ltimes \bar{l})=a d_{\nabla h^{(t)}(\bar{l}, \bar{a})_{-}}^{*}(\bar{a} \ltimes \bar{l}), \quad \frac{\partial}{\partial y}(\bar{a} \ltimes \bar{l})=a d_{\nabla h(y)(\bar{l}, \bar{a})_{-}}(\bar{a} \ltimes \bar{l})
$$

for evolution parameters $y, t \in R$. 
As in Section 3 the presented above construction of Hamiltonian flows on the adjoint space $\overline{\mathcal{G}}^{*}$ can be generalized proceeding to the point product $\overline{\mathcal{G}}^{\Phi^{1}}:=\prod_{z \in \mathbb{S}^{1}} \overline{\mathcal{G}}$ of the holomorphic Lie algebra $\overline{\mathcal{G}}$ endowed with the central extension, generated by a two-cocycle $\omega_{2}: \overline{\mathcal{G}} \times \overline{\mathcal{G}} \rightarrow \mathbb{C}$, where

$$
\omega_{2}\left(\bar{a}_{1} \ltimes \bar{l}_{1}, \bar{a}_{2} \ltimes \bar{l}_{2}\right):=\int_{\mathbb{S}^{1}}\left[\left(\bar{l}_{1}, \partial \bar{a}_{2} / \partial z\right)_{1}-\left(\bar{l}_{2}, \partial \bar{a}_{1} / \partial z\right)_{1}\right]
$$

for any pair of elements $\bar{a}_{1} \ltimes \bar{l}_{1}, \bar{a}_{2} \ltimes \bar{l}_{2} \in \overline{\mathcal{G}}$. The resulting $R$-deformed Lie-Poisson bracket (18) for any smooth functionals $h, f \in D\left(\overline{\mathcal{G}}^{*}\right)$ on the adjoint space $\overline{\mathcal{G}}^{*}$ to the centrally extended loop Lie algebra $\overline{\mathcal{G}}:=\overline{\mathcal{G}} \oplus \mathbb{C}$ becomes equal to

$$
\begin{aligned}
\{h, f\}_{\mathcal{R}}:= & \left(\bar{a} \ltimes \bar{l}_{,}[\nabla h(\bar{l}, \bar{a}), \nabla f(\bar{l}, \bar{a})]_{\mathcal{R}}\right) \\
& +\omega_{2}(\mathcal{R} \nabla h(\bar{l}, \bar{a}), \nabla f(\bar{l}, \bar{a}))+\omega_{2}(\nabla h(\bar{l}, \bar{a}), \mathcal{R} \nabla f(\bar{l}, \bar{a})) .
\end{aligned}
$$

The corresponding Casimir functionals $h^{(p)} \in I\left(\overline{\mathcal{G}}^{*}\right)$ for specially chosen $p \in \mathbb{Z}_{+}$, are defined with respect to the standard Lie-Poisson bracket as

$$
\left\{h^{(p)}, f\right\}:=\left(\bar{a} \ltimes \bar{l},\left[\nabla h^{(p)}(\bar{l}, \bar{a}), \nabla f(\bar{l}, \bar{a})\right]\right)+\omega_{2}\left(\nabla h^{(p)}(\bar{l}, \bar{a}), \nabla f(\bar{l}, \bar{a})\right)=0
$$

for all smooth functionals $f \in D\left(\overline{\mathcal{G}}^{*}\right)$. Based on the equality (21) one easily finds that the gradients $\nabla h^{(p)} \in \overline{\mathcal{G}}$ of the Casimir functionals $h^{(p)} \in I\left(\overline{\mathcal{G}}^{*}\right), p \in \mathbb{Z}_{+}$, satisfy the following equations:

$$
\left[\nabla h_{\bar{l}}, \bar{a}\right]-\frac{\partial}{\partial z} \nabla h_{\bar{l}}=0, \quad a d_{\nabla h_{\bar{l}}}^{*} \bar{l}-a d_{\bar{a}}^{*} \nabla h_{\bar{a}}-\frac{\partial}{\partial z} \nabla h_{\bar{a}}=0
$$

for a chosen element $\bar{a} \ltimes \bar{l} \in \overline{\mathcal{G}}^{*}$. Making use of the suitable Casimir functionals $h^{(y)}, h^{(t)} \in$ $I\left(\overline{\mathcal{G}}^{*}\right)$, one can construct, making use of (47), the following commuting Hamiltonian flows on the adjoint space $\overline{\mathcal{G}}^{*}$ :

$$
\frac{\partial}{\partial y}(\bar{a} \ltimes \bar{l})=\left\{\bar{a} \ltimes \bar{l}, h^{(y)}\right\}_{\mathcal{R}}, \quad \frac{\partial}{\partial t}(\bar{a} \ltimes \bar{l})=\left\{\bar{a} \ltimes \bar{l}, h^{(t)}\right\}_{\mathcal{R}},
$$

which are equivalent to the evolution equations

$$
\frac{\partial}{\partial y} \bar{a}=-\left[\nabla h_{\bar{l},+}^{(y)}, \bar{a}\right]+\frac{\partial}{\partial z} \nabla h_{\bar{l},+}^{(y)}, \quad \frac{\partial}{\partial t} \bar{a}=-\left[\nabla h_{\bar{l},+}^{(t)}, \bar{a}\right]+\frac{\partial}{\partial z} \nabla h_{\bar{l},+}^{(t)}
$$

and

$$
\begin{gathered}
\frac{\partial}{\partial y} \bar{l}=-a d_{\nabla h_{\bar{l},+}^{(y)}}^{*} \bar{l}+a d_{\bar{a}}^{*}\left(\nabla h_{\bar{a}_{,+}}^{(y)}\right)+\frac{\partial}{\partial z} \nabla h_{\bar{a}_{t^{+}}}^{(y)} \\
\frac{\partial}{\partial t} \bar{l}=-a d_{\nabla h_{l_{+}}^{(t)}}^{*} \bar{l}+a d_{\bar{a}}^{*}\left(\nabla h_{\bar{a}_{,+}}^{(t)}\right)+\frac{\partial}{\partial z} \nabla h_{\bar{a}_{{ }_{+}}}^{(t)} .
\end{gathered}
$$

The commutativity condition for these flows is split into two equations

$$
\left[\nabla h_{\bar{l},+}^{(y)}, \nabla h_{\bar{l},+}^{(t)}\right]-\frac{\partial}{\partial t} \nabla h_{\bar{l},+}^{(y)}+\frac{\partial}{\partial y} \nabla h_{\bar{l},+}^{(t)}=0,
$$

and

$$
\begin{gathered}
\frac{\partial \bar{P}}{\partial z}+a d_{\bar{a}}^{*} \bar{P}=0, \\
\bar{P}=a d_{\nabla h_{\bar{l},+}^{(y)}}^{*}\left(\nabla h_{\bar{a},+}^{(t)}\right)-a d_{\nabla h_{\bar{l},+}^{(t)}}^{*}\left(\nabla h_{\bar{a}_{,+}}^{(y)}\right)-\frac{\partial}{\partial t} \nabla h_{\bar{a},+}^{(y)}+\frac{\partial}{\partial y} \nabla h_{\bar{a},+}^{(t)}
\end{gathered}
$$

for any $\bar{a} \ltimes \bar{l} \in \bar{G}$. The obtained above results one can be formulated as the following proposition. 
Proposition 7. The Hamiltonian flows (48) on the adjoint space $\overline{\mathcal{G}}^{*}$ generate the separately commuting evolution equations (49) and (50). The evolution equations (49) give rise to the Lax type compatibility condition (51), being equivalent to some system of nonlinear heavenly type equations in partial derivatives. Moreover, the system of evolution equations (49) can be considered as the compatibility condition for the following set of linear vector equations

$$
\frac{\partial \psi}{\partial y}+\nabla h_{\bar{l},+}^{(y)} \psi=0, \quad \frac{\partial \psi}{\partial z}+\langle a, \partial / \partial \mathbf{x}\rangle \psi=0, \quad \frac{\partial \psi}{\partial t}+\nabla h_{\bar{l},+}^{(t)} \psi=0
$$

for all $(y, t, z ; x) \in\left(\mathbb{R}^{2} \times \mathbb{S}^{1}\right) \times \mathbb{T}^{n}$ and a function $\psi \in C^{2}\left(\left(\mathbb{R}^{2} \times \mathbb{C} \times \mathbb{S}^{1}\right) \times \mathbb{T}^{n} ; \mathbb{C}\right)$.

\subsection{Example: the generalized Mikhalev-Pavlov heavenly type system}

Let a seed element $\bar{a} \ltimes \bar{l} \in \overline{\mathcal{G}}^{*}$ be chosen as

$$
\bar{a} \ltimes \bar{l}=\left(\left(u_{x}-\lambda\right) \partial / \partial x+v_{x} \partial / \partial \lambda\right) \ltimes\left(w_{x} d x+\eta_{x} d \lambda\right),
$$

where $u, v, w, \eta \in C^{2}\left(\mathbb{R}^{2} \times\left(\mathbb{S}^{1} \times \mathbb{T}^{1}\right) ; \mathbb{R}\right)$. The asymptotic splits for the components of the gradients of the corresponding Casimir functionals $h^{(p)} \in I\left(\overline{\mathcal{G}}^{*}\right), p \in \mathbb{Z}_{+}$, as $|\lambda| \rightarrow \infty$ have the following forms:

$$
\begin{aligned}
& \nabla h_{\tilde{l}} \simeq \lambda^{p}\left(\begin{array}{c}
1-u_{x} \lambda^{-1}+\left(-u_{z}+(p-1) v\right) \lambda^{-2}+\left(u_{y}+(p-2)\left(-u_{x} v+\varkappa\right)\right) \lambda^{-3}+\ldots \\
-v_{x} \lambda^{-1}-v_{z} \lambda^{-2}+\left(v_{y}-(p-2) v_{x} v\right) \lambda^{-3}+\ldots
\end{array}\right), \\
& \nabla h_{\tilde{a}} \simeq \lambda^{p}\left(\begin{array}{c}
-w_{x} \lambda^{-1}-\left(\eta_{z}+(p-1) w\right) \lambda^{-2}+\left(\eta_{y}-(p-2)\left(-u_{x} w+v \eta_{x}+\omega\right)\right) \lambda^{-3}+\ldots
\end{array}\right),
\end{aligned}
$$

where $p \in \mathbb{Z}_{+}$and

$$
\varkappa_{x}=v_{z}+u_{x} v_{x}, \quad \omega_{x}=w_{z}-u_{x} w_{x}-v_{x} \eta_{x}
$$

In the case when

$$
\begin{aligned}
\nabla h_{\tilde{l},+}^{(y)} & :=\left(\begin{array}{c}
\lambda^{2}-u_{x} \lambda+\left(-u_{z}+v\right) \\
-v_{x} \lambda-v_{z}
\end{array}\right), \\
\nabla h_{\tilde{a},+}^{(y)} & :=\left(\begin{array}{c}
-w_{x} \lambda-w_{z} \\
-\eta_{x} \lambda-\left(\eta_{z}+w\right)
\end{array}\right),
\end{aligned}
$$

and

$$
\begin{aligned}
& \nabla h_{\tilde{l},+}^{(t)}:=\left(\begin{array}{c}
\lambda^{3}-u_{x} \lambda^{2}+\left(-u_{z}+2 v\right) \lambda+\left(u_{y}-u_{x} v+\varkappa\right) \\
-v_{x} \lambda^{2}-v_{z} \lambda+\left(v_{y}-v_{x} v\right)
\end{array}\right), \\
& \nabla h_{\tilde{a},+}^{(t)}:=\left(\begin{array}{c}
-w_{x} \lambda^{2}-w_{z} \lambda+\left(w_{y}-(w v)_{x}\right) \\
-\eta_{x} \lambda^{2}-\left(\eta_{z}+2 w\right) \lambda+\left(\eta_{y}+u_{x} w-v \eta_{x}-\omega\right)
\end{array}\right),
\end{aligned}
$$


the compatibility condition of the Hamiltonian vector flows (48) leads to the system of evolution equations:

$$
\begin{aligned}
u_{z t}+u_{y y} & =-u_{y} u_{z x}+u_{z} u_{x y}-u_{x y} v-u_{z z} v-\varkappa u_{x z} \\
v_{z t}+v_{y y} & =v v_{x}^{2}-v_{z}^{2}-v v_{x y}-v v_{z z}-u_{y} v_{x z}+u_{z} v_{x y}-u_{z} v_{x}^{2}-\varkappa v_{x z} \\
-u_{x y}-u_{z z} & =u_{x} u_{x z}-u_{z} u_{x x}+u_{x x} v \\
-v_{x y}-v_{z z} & =v_{x}^{2}+v_{x x} v+u_{x} v_{x z}-u_{z} v_{x x} \\
-u_{x t}+u_{y z} & =-u_{x} u_{x y}+u_{y} u_{x x}+u_{x z} v+u_{x x} \varkappa \\
-v_{x t}+v_{y z} & =-u_{x} v_{x y}+u_{y} v_{x x}+u_{x} v_{x}^{2}+v_{x z} v+\varkappa v_{x x}+2 v_{x} v_{z} .
\end{aligned}
$$

Under the constraint $v=0$ one obtains the set of equations (29)-(31). Thus, the following proposition holds.

Proposition 8. The constructed system of heavenly type equations (54) and (53) has the LaxSato vector field representation (51) with the "spectral" parameter $\lambda \in \mathbb{C}$, which is related with element $\bar{a} \ltimes \bar{l} \in \overline{\mathcal{G}}^{*}$ in the form (52).

\section{ACKNOWLEDGEMENTS}

The authors cordially thank M. Błaszak, J. Ciesliński and B. Szablikowski for useful discussions of the results during the XI-th "Integrable Systems" Symposium, held on June 29-30, 2018 in Poznań, Poland. They also are very appreciated to Gerald Goldin and Anatol Odziewicz for fruitful and instructive comments and remarks during the XXXVII Workshop on Geometric Methods in Physics during the XXVIII International Workshop on "Geometry in Physics", held on June 30-July 07, 2019 in Białowieża, Poland.

\section{REFERENCES}

[1] Abraham R., Marsden J. Foundations of Mechanics. Benjamin Cummings, New York, 1994.

[2] Alekseev A., Malkin A.Z. Symplectic structure of the modili space of flat connection on a Riemann surface. Comm. Math. Phys. 1995, 169, 99-119.

[3] Alonso L.M., Shabat A.B. Hydrodynamic reductions and solutions of a universal hierarchy. Theoret. and Math. Phys. 2004, 104 (1), 1073-1085. doi:10.1023/B:TAMP.0000036538.41884.57

[4] Arnold V.I. Mathematical Methods of Classical Mechanics. Springer, New York, 1978.

[5] Arnold V.I. Sur la geometrie differerentielle des groupes de Lie de dimension infinie et ses applications a l'hydrodynamique des fluides parfaits. Ann. Inst. Fourier (Grenoble) 1966, 16, 319-361.

[6] Arnold V.I., Khesin B.A. Topological methods in hydrodynamics. Springer, New York, 1998.

[7] Artemovych O.D., Balinsky A.A., Blackmore D., Prykarpatski A.K. Reduced Pre-Lie Algebraic Structures, the Weak and Weakly Deformed Balinsky-Novikov Type Symmetry Algebras and Related Hamiltonian Operators. Symmetry 2018, 10 (11), 601. doi:10.3390/sym10110601

[8] Artemovych O.D., Blackmore D., Prykarpatski A.K. Examples of Lie and Balinsky-Novikov algebras related to Hamiltonian operators. Topol. Algebra Appl. 2018, 6, 43-52. doi:10.1515/taa-2018-0005 
[9] Artemovych O.D., Blackmore D., Prykarpatski A.K. Poisson brackets, Novikov-Leibniz structures and integrable Riemann hydrodynamic systems. J. Nonlinear Math. Phys. 2017, 24 (1), 41-72. doi:10.1080/14029251.2016.1274114

[10] Audin M. Lectures on gauge theory and integrable systems. In: Hurtubise J., Lalonde F. (Eds.) Gauge Theory and Symplectic Geometry, Kluwer, 1-48, 1997.

[11] Blackmore D., Prykarpatsky A.K., Samoylenko V.H. Nonlinear Dynamical Systems of Mathematical Physics. World Scientific Publisher Co. Pte. Ltd., Hackensack, USA, 2011. doi:10.1142/7960

[12] Blackmore B., Prykarpatsky Y., Golenia J., Prykapatski A. Hidden Symmetries of Lax Integrable Nonlinear Systems. Applied Mathematics 2013, 4 (10), 95-116. doi:10.4236/am.2013.410A3013

[13] Błaszak M. Classical R-matrices on Poisson algebras and related dispersionless systems. Phys. Lett. A 2002, 297 (3-4), 191-195. doi:10.1016/S0375-9601(02)00421-8

[14] Błaszak M. Multi-Hamiltonian theory of dynamical systems. Springer, Berlin, 1998.

[15] Błaszak M., Szablikowski B.M. Classical R-matrix theory of dispersionless systems: II. $(2+1)$ dimension theory. J. Phys. A: Math. Gen. 2002, 35 (48), 10325. doi:10.1088/0305-4470/35/48/309

[16] Bogdanov L.V., Dryuma V.S., Manakov S.V. Dunajski generalization of the second heavenly equation: dressing method and the hierarchy. J. Phys. A: Math. Theor. 2007, 40 (48), 14383-14393.

[17] Bogdanov L.V., Pavlov M.V. Linearly degenerate hierarchies of quasiclassical SDYM type. J. Math. Phys. 2017, 58, 093505. doi:10.1063/1.5004258

[18] Doubrov B., Ferapontov E.V. On the integrability of symplectic Monge-Ampère equations. J. Geom. Phys. 2010, 60 (10), 1604-1616.

[19] Doubrov B., Ferapontov E.V., Kruglikov B., Novikov V.S. On integrability in Grassmann geometries: integrable systems associated with fourfolds in Gr(3,5). Proc. Lond. Math. Soc. (3) 2018, 116 (5), 1269-1300. doi:10.1112/plms.12114

[20] Dunajski M. Anti-self-dual four-manifolds with a parallel real spinor. Proc. Roy. Soc. Edinburgh Sect. A 2002, 458, 1205-1222. doi:10.1098/rspa.2001.0918

[21] Dunajski M., Mason L.J., Tod P. Einstein-Weyl geometry, the dKP equation and twistor theory. J. Geom. Phys. 2001, 37 (1-2), 63-93.

[22] Ferapontov E.V., Kruglikov B. Dispersionless integrable systems in 3D and Einstei-Weyl geometry. J. Differential Geom. 2012, 97 (2), 215-254. doi:10.4310/jdg/1405447805

[23] Ferapontov E.V., Moss J. Linearly degenerate PDEs and quadratic line complexes. Comm. Anal. Geom. 2015, 23 (1), 91-127. doi:10.4310/CAG.2015.v23.n1.a3

[24] Godbillon C. Geometrie Differentielle et Mecanique Analytique. Hermann Publ., Paris, 1969.

[25] Hentosh O.Ye., Prykarpatsky Ya.A., Blackmore D., Prykarpatski A.K. Lie-algebraic structure of Lax-Sato integrable heavenly equations and the Lagrange-d'Alembert principle. J. Geom. Phys. 2017, 120, 208-227.

[26] Holm D., Kupershmidt B. Poisson structures of superfluids. Phys. Lett. 1982, 91 (A), 425-430. doi: 10.1016/j.geomphys.2017.06.003

[27] Holm D., Marsden J., Ratiu T., Weinstein A. Nonlinear stability of fluid and plasma equilibria. Phys. Rep. 1985, $123(1-2), 1-116$.

[28] Kambe T. Geometric theory of fluid flows and dynamical systems. Fluid Dyn. Res. 2002, 30, 331-378.

[29] Kulish P.P. An analogue of the Korteweg-de Vries quation for the superconformal algebra. J. Math. Sci. 1988, 41 (2), 970-975. doi:10.1007/BF01247091

[30] Kupershmidt B.A., Ratiu T. Canonical Maps Between Semidirect Products with Applications to Elasticity and Superfluids. Commun. Math. Phys. 1983, 90, 235-250. doi:10.1007/BF01205505 
[31] Kuznetsov E.A., Mikhailov A.V. On the topological meaning of canonical Clebsch variables. Phys. Lett. A 1980, 77 (1), 37-38. doi:10.1016/0375-9601(80)90627-1

[32] Manakov S.V., Santini P.M. On the solutions of the second heavenly and Pavlov equations. J. Phys. A 2009, 42 (40), 404013.

[33] Marsden J., Ratiu T., Schmid R., Spencer R., Weinstein A. Hamiltonian systems with symmetry, coadjoint orbits, and plasma physics. Atti Acad. Sci. Torino Cl. Sci. Fis. Math. Natur. 117, 1983, 289-340.

[34] Marsden J., Ratiu T., Weinstein A. Reduction and Hamiltoninan structures on duals of semidirect product Lie algebras. Contemp. Math. 1984, 28, 55-100.

[35] Marsden J., Weinstein A. Reduction of symplectic manifolds with symmetry. Rep. Math. Phys. 1974, 5, 121-130.

[36] Mikhalev V.G. On the Hamiltonian formalism for Korteweg-de Vries type hierarchies. Funct. Anal. Appl. 1992, 26 (2), 140-142. doi:10.1007/BF01075282

[37] Misiolek G. A shallow water equation as a geodesic flow on the Bott-Virasoro group. J. Geom. Phys. 1998, 24 (3), 203-208.

[38] Ovsienko V. Bi-Hamilton nature of the equation $u_{t x}=u_{x y} u_{y}-u_{y y} u_{x}$. arXiv:0802.1818v1

[39] Ovsienko V., Roger C. Looped Cotangent Virasoro Algebra and Non-Linear Integrable Systems in Dimension $2+1$. Commun. Math. Phys. 2007, 273, 357-378.

[40] Pavlov M.V. Integrable hydrodynamic chains. J. Math. Phys. 2003, 44 (9), 4134-4156.

[41] Plebański J.F. Some solutions of complex Einstein equations. J. Math. Phys. 1975, 16 (12), 2395-2402.

[42] Pressley A., Segal G. Loop groups. Clarendon Press, London, 1986.

[43] Prykarpatski A.K., Hentosh O.Ye., Prykarpatsky Ya.A. Geometric Structure of the Classical Lagranged'Alambert Principle and its Application to Integrable NonlinearDynamical Systems. Mathematics 2017, 5 (75), 1-20. doi:10.3390/math5040075

[44] Prykarpatsky A.K., Mykytyuk I.V. Algebraic integrability of nonlinear dynamical systems on manifolds: classical and quantum aspects. Kluwer Academic Publishers, Netherlands, 1998.

[45] Reyman A.G., Semenov-Tian-Shansky M.A. Integrable Systems. The Computer Research Institute Publ., Moscow-Izhvek, 2003. (in Russian)

[46] Schief W.K. Self-dual Einstein spaces and a discrete Tzitzeica equation. A permutability theorem link. In: Clarkson P.A., Nijhoff F.W. (Eds.) Symmetries and Integrability of Difference Equations, London Mathematical Society, Lecture Note Series 255, Cambridge University Press, 1999, 137-148.

[47] Schief W.K. Self-dual Einstein spaces via a permutability theorem for the Tzitzeica equation. Phys. Lett. A 1996, 223 (25), 55-62.

[48] Semenov-Tian-Shansky M.A. What is a classical R-matrix? Funct. Anal. Appl. 1983, 17, 259-272. doi: 10.1007/BF01076717

[49] Sergyeyev A., Szablikowski B.M. Central extensions of cotangent universal hierarrchy: (2+1)-dimensional biHamiltonian systems Phys. Lett. A 2008, 372 (47), 7016-7023. doi:10.1016/j.physleta.2008.10.020

[50] Strachan I.A.B., Szablikowski B.M. Novikov algebras and a classification of multicomponent Camassa-Holm equations. Stud. Appl. Math. 2014, 133, 84-117. doi:10.1111/sapm.12040

[51] Szablikowski B. Hierarchies of Manakov-Santini Type by Means of Rota-Baxter and Other Identities. SIGMA Symmetry Integrability Geom. Methods Appl. 2016, 12, 022, 14 pages. doi:10.3842/SIGMA.2016.022

[52] Takasaki K., Takebe T. Integrable Hierarchies and Dispersionless Limit. Rev. Math. Phys. 1995, 7 (05), $743-808$. 
[53] Takasaki K., Takebe T. SDiff(2) Toda equation - Hierarchy, Tau function, and Symmetries. Lett. Math. Phys. 1991, 23 (3), 205-214. doi:10.1007/BF01885498

[54] Takhtadjian L.A., Faddeev L.D. Hamiltonian Approach in Soliton Theory. Springer, Berlin-Heidelberg, 1987.

[55] Warner F.W. Foundations of Diffderentiable Manifolds and Lie Groups. Springer, Ney York, 1983.

[56] Weinstein A. Sophus Lie and symplectic geometry. Expos. Math. 1983, 1, 95-96.

[57] Weinstein A. The local structure of Poisson manifolds. J. Differential Geom. 1983, 18, 523-557.

Received 22.05.2020

Гентош О.Є., Балінський О.А., Прикарпатський А.К. Узагальнені центрально розщирені Аі-алгебрайчні структури та асоцйовані інтегровні рівняння небесного типу // Карпатські матем. публ. — 2020. — T.12, №1. — С. 242-264.

Вивчаються центрально розширені $\Lambda$-алгебраїчні структури та аоційовані інтегровні рівняння небесного типу як потоків на орбітах коприєднаної дії півпрямої суми алгебри векторних полів на торі та ії спряженого простору. Показано, що ц потоки породжують сумісні векторні поля типу Аакса-Сато, з якими тісно пов'язана нескінченна ієрархія законів збереження, породжених відповідними інваріантами Казіміра. Наводено типові приклади таких рівнянь і детально продемонстрована їх інтегровність в межах запропоновоної схеми. Як приклади ми отримали та описали нові багатовимірні інтегровні узагальнення бездисперсійних рівнянь Михальова-Павлова та Алонсо-Шабата, для котрих генераторні елементи мають особливу факторизовану структуру, шо дозволяє поширити їх на випадок довільного виміру.

Ключові слова і фрази: рівняння небесного типу, інтегровність за Ааксом, динамічна система Гамільтона, дифеоморфізми тора, алгебра $\Lambda$ і петель, центральне розширення, $\Lambda$ i-алгебраїчна схема, інваріанти Казіміра, структура $\Lambda$ i-Пуассона, $R$-структура, рівняння Міхальова-Павлова. 